\title{
Joint Source and Channel Coding for Image Transmission over Noisy Channels with Feedback
}

\author{
by \\ Jinshi Qiu \\ A thesis submitted to the \\ Faculty of Graduate Studies and Research \\ in partial fulfillment of the requirements for the degree of \\ Master of Applied Science \\ in Electrical Engineering
}

Ottawa-Carleton Institute for Electrical and Computer Engineering

Department of Systems and Computer Engineering

Carleton University

Ottawa, Ontario, Canada

May 2008

(C) Copyright 2008, Jinshi Qiu 


$\begin{array}{ll}\begin{array}{l}\text { Library and } \\ \text { Archives Canada }\end{array} & \begin{array}{l}\text { Bibliothèque et } \\ \text { Archives Canada }\end{array} \\ \begin{array}{l}\text { Published Heritage } \\ \text { Branch }\end{array} & \begin{array}{l}\text { Direction du } \\ \text { Patrimoine de l'édition }\end{array} \\ \begin{array}{l}\text { 395 Wellington Street } \\ \text { Ottawa ON K1A 0N4 } \\ \text { Canada }\end{array} & \begin{array}{l}\text { 395, rue Wellington } \\ \text { Ottawa ON K1A 0N4 } \\ \text { Canada }\end{array}\end{array}$

Your file Votre référence ISBN: 978-0-494-40644-1 Our file Notre référence ISBN: 978-0-494-40644-1

NOTICE:

The author has granted a nonexclusive license allowing Library and Archives Canada to reproduce, publish, archive, preserve, conserve, communicate to the public by telecommunication or on the Internet, loan, distribute and sell theses worldwide, for commercial or noncommercial purposes, in microform, paper, electronic and/or any other formats.

The author retains copyright ownership and moral rights in this thesis. Neither the thesis nor substantial extracts from it may be printed or otherwise reproduced without the author's permission.
AVIS:

L'auteur a accordé une licence non exclusive permettant à la Bibliothèque et Archives Canada de reproduire, publier, archiver, sauvegarder, conserver, transmettre au public par télécommunication ou par l'Internet, prêter, distribuer et vendre des thèses partout dans le monde, à des fins commerciales ou autres, sur support microforme, papier, électronique et/ou autres formats.

L'auteur conserve la propriété du droit d'auteur et des droits moraux qui protège cette thèse. $\mathrm{Ni}$ la thèse ni des extraits substantiels de celle-ci ne doivent être imprimés ou autrement reproduits sans son autorisation.
In compliance with the Canadian Privacy Act some supporting forms may have been removed from this thesis.

While these forms may be included in the document page count, their removal does not represent any loss of content from the thesis.
Conformément à la loi canadienne sur la protection de la vie privée, quelques formulaires secondaires ont été enlevés de cette thèse.

Bien que ces formulaires aient inclus dans la pagination, il n'y aura aucun contenu manquant.

\section{Canada}




\section{Abstract}

We propose solutions to the rate allocation problem of progressive image transmission over noisy channels with feedback. The scenario we are considering is the feedback constraint on each packet, which means the number of feedback bits on each packet is upper bounded by a fixed given value. Both rate-optimal and distortion-optimal solutions are investigated. Simulation results show that the proposed solutions outperform the previously published results and can closely achieve the performance of ARQ scheme with infinite number of feedback bits as the number of feedback bits increases. Although the simulations are performed using JPEG2000 source coder and rate-compatible LDPC channel codes, the solutions presented in this thesis are applicable to any progressive source coding scheme, any family of rate-compatible channel codes, and any channel model as long as the distortion-rate function of the source and the error probabilities of the channel codes are available. 


\section{Acknowledgements}

I would like to take this opportunity to express my deep gratitude to all people who offered their help in the completion of my thesis.

My gratitude first goes towards my thesis supervisor, Professor Amir H. Banihashemi, for his insightful suggestions, patient guidance and continuous encouragement in my research work.

I want to thank my examination committee, Professor Eric Dubois (External Examiner from SITE, University of Ottawa), Professor Roshdy Hafez (Chair) and Professor Ioannis Lambadaris for their good comments and suggestions on to my thesis.

Many thanks also goes to all my friends and colleagues at our research group and laboratory, especially Hua Xiao, Chantong Lam, Xiaoning Li, Xiaosong Lu, Jie Zhu, Chenjuan Zhou, Jie Liu, Haishan Wang, Sebastian S. Szyszkowicz, and Saad Al-Ahmadi, for their passionate help, good suggestions, enjoyable soccer games and amusing chats.

Most importantly, I would like to acknowledge my beloved wife, Xi Zhou, for her understanding, support, and continuous love. I could not take care of her during my graduate study at Carleton University. I am forever indebted to her.

Jinshi Qiu

May 12, 2008

Ottawa, Canada 


\section{Table of Contents}

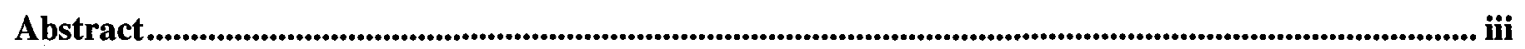

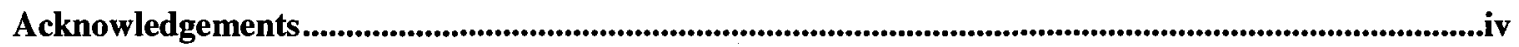

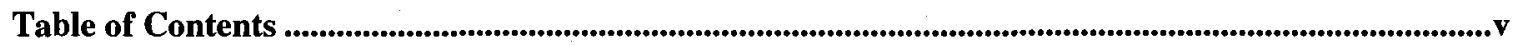

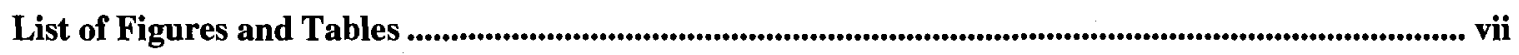

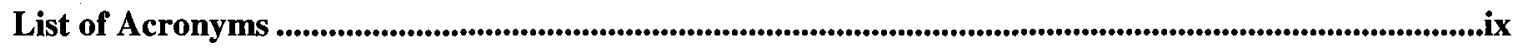

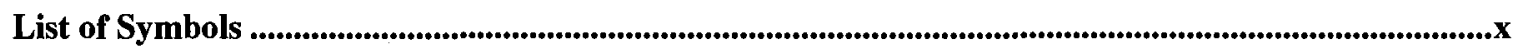

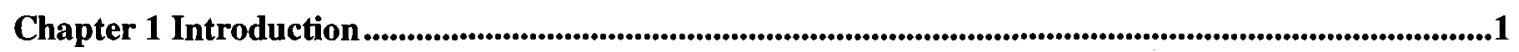

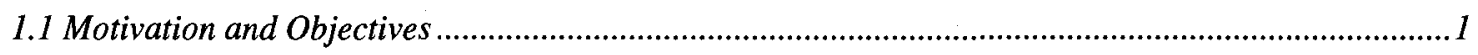

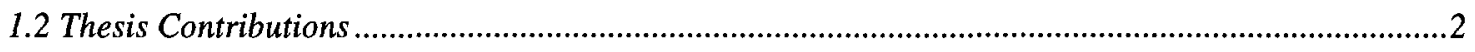

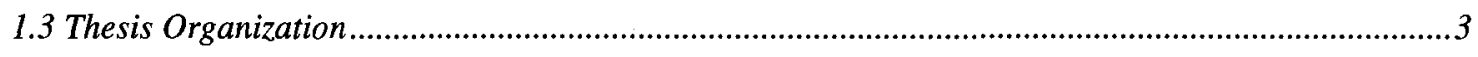

Chapter 2 Background and Literature Review ..........................................................................................5

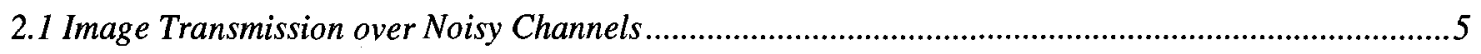

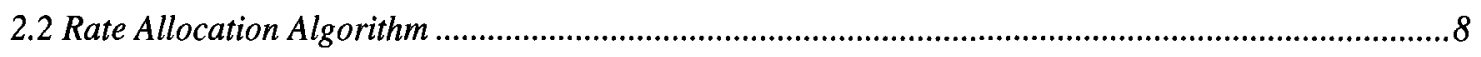

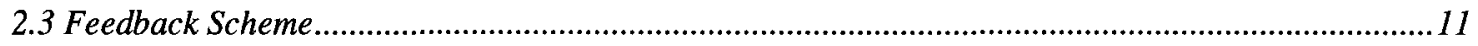

Chapter 3 Rate Allocation for Feedback Channels with a Constraint on the Number of Feedback Bits

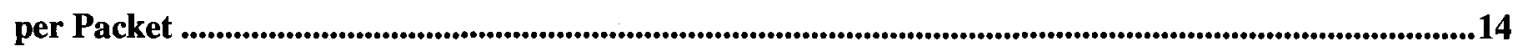

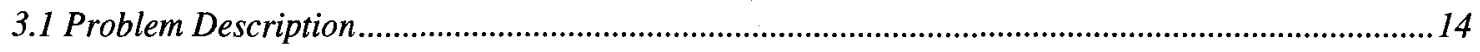

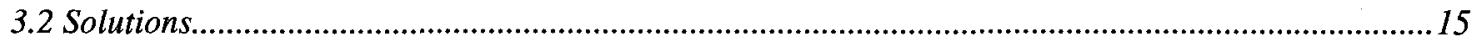

3.2.1 Solution 1: Rate-optimal Solution Proposed by Chande et al ....................................................15

3.2.2 Solution 2: Our Proposed Rate-optimal Solution ......................................................................17

3.2.3 Solution 3: Proposed Distortion-optimal Solution .......................................................................23 
3.4 Comparison of the Proposed Solutions with Solution 1 ...............................................................27

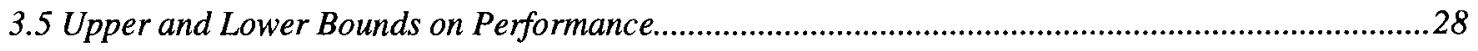

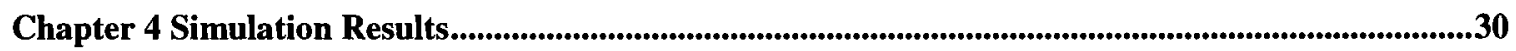

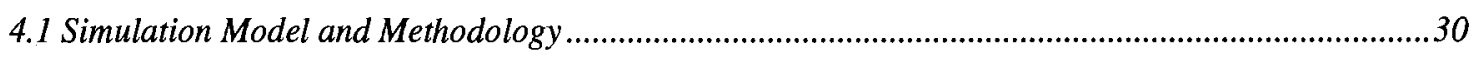

4.1.1 Correlated Rayleigh Fading Channel with SI: Vehicle Speed V=6 km/h...................................34

4.1.2 Correlated Rayleigh Fading Channel with SI: Vehicle Speed V=50 km/h................................35

4.1.3 Correlated Rayleigh Fading Channel with SI: Vehicle Speed V=120 km/h.............................36

4.2 The Number of Candidate Policies Left in $\eta$ after Using the Simplification Measures........................37

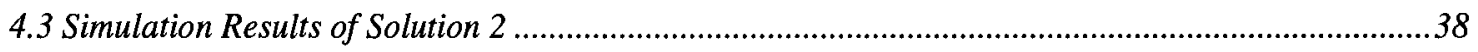

4.3.1 Simulation Results of Solution 2 at Vehicle Speed $=6 \mathrm{~km} / \mathrm{h}$..................................................... 39

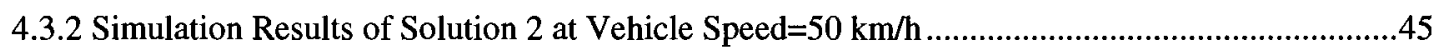

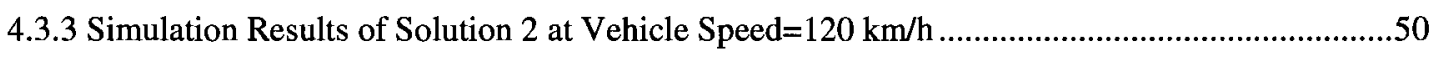

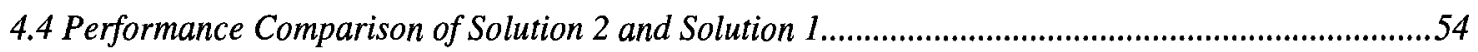

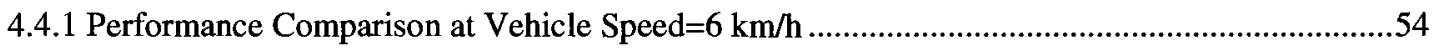

4.4.2 Performance Comparison at Vehicle Speed $=50 \mathrm{~km} / \mathrm{h}$........................................................5

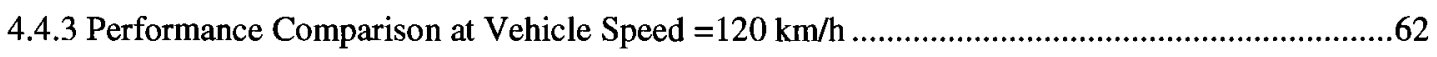

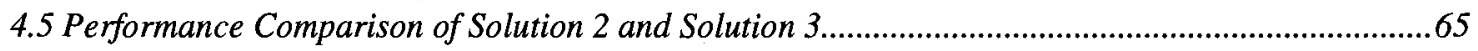

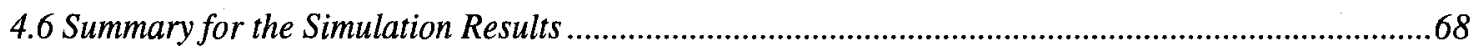

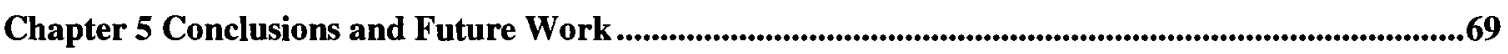

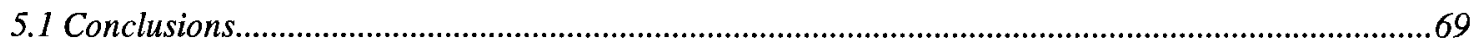

5.2 Future Work

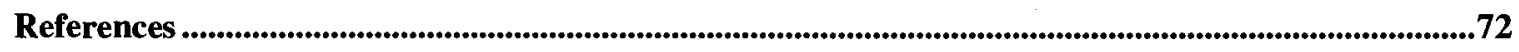




\section{List of Figures and Tables}

Figure 2-1 Block diagram of image transmission over a noisy channel.

Figure 2-2 Progressive image transmission

Figure 2-3 Block diagram of a transmission scheme with the rate allocation algorithm

Figure 2-4 Block diagram of image transmission over noisy channel with HARQ.

Table 4-1 Packet error probabilities of channel codes for correlated Rayleigh fading channel with SI, V=6 km/h

Table 4-2 Packet error probabilities of channel codes for correlated Rayleigh fading channel with SI, V=50 km/h .35

Table 4-3 Packet error probabilities of channel codes for correlated Rayleigh fading channel with SI, V=120 km/h . 36

Table 4-4 The number of candidate policies in $\eta$ before and after using the simplification measures of Section 3.3

Figure 4-1 Performance of Solution 2: $V=6 \mathrm{~km} / \mathrm{h}, \mathrm{SNR}=10,14$, and $20 \mathrm{~dB}$

Figure 4-2 Performance of Solution 2: $V=6 \mathrm{~km} / \mathrm{h}, \mathrm{SNR}=10 \mathrm{~dB}$ 42

Figure 4-3 Performance of Solution 2: $V=6 \mathrm{~km} / \mathrm{h}, \mathrm{SNR}=14 \mathrm{~dB}$

Figure 4-4 Performance of Solution 2: $V=6 \mathrm{~km} / \mathrm{h}, \mathrm{SNR}=20 \mathrm{~dB}$ .44

Figure 4-5 Performance of Solution 2: V=50 km/h, $S N R=10,14$, and $20 \mathrm{~dB}$ 46

Figure 4-6 Performance of Solution 2: V=50 km/h, SNR=10 dB. 47 
Figure 4-7 Performance of Solution 2: $V=50 \mathrm{~km} / \mathrm{h}, \mathrm{SNR}=14 \mathrm{~dB}$

Figure 4-8 Performance of Solution 2: V=50 km/h, SNR=20 dB

Figure 4-9 Performance of Solution 2: $\mathrm{V}=120 \mathrm{~km} / \mathrm{h}, \mathrm{SNR}=10$, 14, and $20 \mathrm{~dB}$

Figure 4-10 Performance of Solution 2: V=120 km/h, SNR=10 dB. .51

Figure 4-11 Performance of Solution 2: V=120 km/h, SNR=14 dB. 52

Figure 4-12 Performance of Solution 2: V=120 km/h, SNR=20 dB.

Table 4-5 Performance comparison of Solution 2 and Solution $1 \mathrm{in} \mathrm{dB}$ : $\mathrm{V}=6 \mathrm{~km} / \mathrm{h}, \mathrm{SNR}=10 \mathrm{~dB} \ldots . . .56$

Figure 4-13 Performance comparison of Solution 2 and Solution 1: $V=6 \mathrm{~km} / \mathrm{h}, \mathrm{SNR}=10 \mathrm{~dB}$ .57

Figure 4-14 Performance comparison of Solution 2 and Solution 1: $V=6 \mathrm{~km} / \mathrm{h}, \mathrm{SNR}=14$ and $20 \mathrm{~dB}$

Table 4-6 Performance comparison of Solution 2 and Solution 1: $V=50 \mathrm{~km} / \mathrm{h}, \mathrm{SNR}=10,14$ and $20 \mathrm{~dB}$

Figure 4-15 Performance comparison of Solution 2 and Solution 1: $V=50 \mathrm{~km} / \mathrm{h}, \mathrm{SNR}=10,14$ and 20

dB. . .61

Table 4-7 Performance comparison of Solution 2 and Solution 1: $V=120 \mathrm{~km} / \mathrm{h}, \mathrm{SNR}=10,14$ and 20

dB.

Figure 4-16 Performance comparison of Solution 2 and Solution 1: V=120 km/h, SNR=10, 14 and 20

dB. . .64

Figure 4-17 Performance comparison of Solution 2 and Solution 3: V=6 km/h, SNR=10, 14 and 20 dB.

Figure 4-18 Performance comparison of Solution 2 and Solution 3: V=6 km/h, SNR=10 dB . .67 


\section{List of Acronyms}

ACK

ARQ

BER

bpp

CRC

EEP

FEC

FPP

HARQ

IRA

JPEG2000

JSCC

LDPC

MSE

NACK

PSNR

$\mathrm{RC}$

RC-IRA

RC-LDPC

$\mathrm{RCPC}$

RCTC

RMSE

SPIHT

UEP

VPP
Acknowledgement

Automatic Repeat Request

Bit Error Rate

bit per pixel

Cyclic Redundancy Check

Equal Error Protection

Forward Error Correction

Fixed-length Packet Problem

Hybrid ARQ

Irregular Repeat Accumulate

Joint Photographic Experts Group 2000

Joint Source and Channel Coding

Low-Density Parity-Check codes

Mean Squared Error

Negative Acknowledgement

Peak Signal to Noise Ratio

Rate-Compatible

Rate-Compatible Irregular Repeat Accumulate

Rate-Compatible Low-Density Parity-Check Codes

Rate-Compatible Punctured Convolutional Codes

Rate-Compatible Turbo Codes

Root Mean Squared Error

Set Partitioning in Hierarchical Trees

Unequal Error Protection

Variable-length Packet Problem 


\section{List of Symbols}

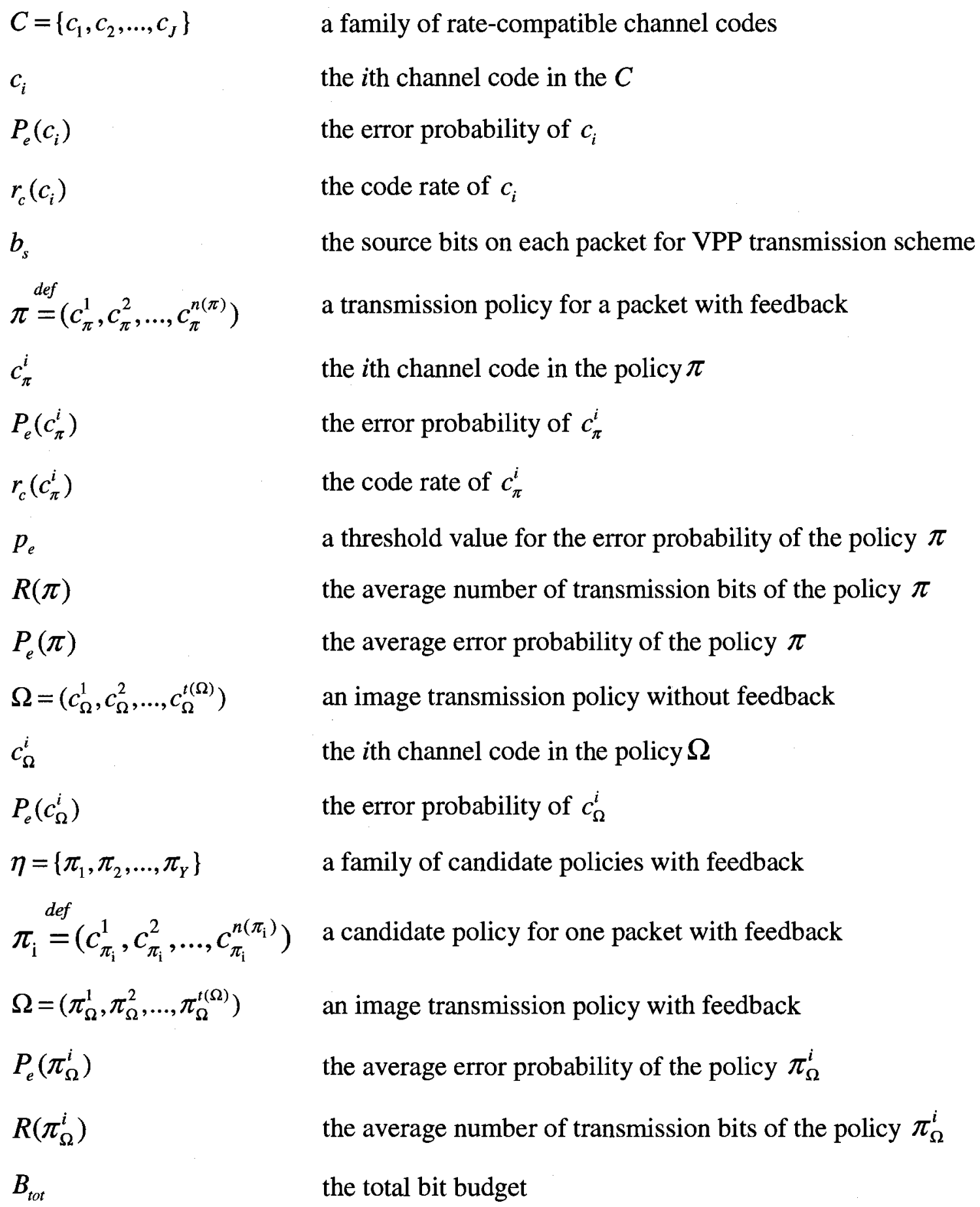


the throughput of Type II hybrid ARQ scheme the number of useful source bits 


\section{Chapter 1 Introduction}

\subsection{Motivation and Objectives}

A progressively coded bitstream is extremely attractive and widely used in image transmission over noisy channels since it allows the receiver to reconstruct the image at different bit rates from the prefixes of the bitstream, providing continually improving approximation to the original image as more bits arrive and are correctly decoded. However, a big drawback of a progressively coded bitsream is that it is highly sensitive to bit errors caused by the channel noise. Therefore, error control techniques are required to protect the bitstream against channel errors when image transmission is on a noisy channel. The forward error correction (FEC) technique can provide near error-free transmission without delay, but it increases the total transmission rate (channel bits) because of the redundancy introduced. On the contrary, the automatic repeat request (ARQ) technique can eliminate channel errors with as few as possible total transmission bits, but it causes delay due to the retransmissions. In some transmission scenarios with a feedback channel available, both the total transmission rate and the number of feedback bits (feedback opportunities) have constraints. For such scenarios, Hybrid ARQ (HARQ), an error control technique combining the advantages of FEC and ARQ, is employed to achieve a good performance under the constraints [4]-[5], [12], [18]-[19]. It is wellknown that joint source and channel coding (JSCC) can achieve a good end-to-end performance when the total number of transmission bits is given [7]-[8]. For JSCC, the 
rate allocation problem, a problem on how to choose the code rates for packet transmission under the constraints of total transmission bits and the number of feedback bits so as to achieve the optimal end-to-end performance, is a key component. One can consider two different constraints on the number of feedback bits. One is the number of feedback bits on each packet is given, and the other is the total number of feedback bits on total packets is given. We refer to the former scenario as feedback constraint on each packet, and refer to the latter scenario as feedback constraint on total packets. The rate allocation problem for the former scenario was first investigated in [21], in which Chande et al. proposed an imprecise approach to choose a sequence of channel codes for the transmission of each packet. Moreover, this approach is rate-optimal [16], which is normally inferior to the distortion-optimal approach [16]. The purpose of this work is to obtain rate-optimal and distortion-optimal rate-allocation solutions for progressive transmission of bit streams on feedback channels. Our main focus is on the rate allocation for the problem of feedback constraint on each packet.

\subsection{Thesis Contributions}

The major contributions of this thesis include:

1. an effective rate-optimal solution to the rate allocation problem with a feedback constraint on each packet;

2. an effective distortion-optimal solution to the rate allocation problem with a feedback constraint on each packet; 
3. an effective approach to reduce the computational complexity of the proposed solutions;

To achieve these contributions, the following novel concepts and equations are introduced:

1. a concept of average error probability for a packet-based transmission policy, which consists of a sequence of channel codes

2. a concept of average number of transmission bits for a packet-based transmission policy, which consists of a sequence of channel codes;

3. a closed-form equation to compute the average error probability for a packetbased transmission policy;

4. a closed-form equation to compute the average number of transmission bits for a packet-based transmission policy.

\subsection{Thesis Organization}

In Chapter 2, we present the background and literature review related to the thesis work. First of all, a general framework for image transmission over noisy channels is illustrated, then some basic concepts including progressive source coding, joint source and channel coding, unequal error protection and rate-compatible codes are described. Secondly, the rate allocation problem, along with the nomenclatures of rate optimality and distortion optimality, are introduced. Finally, the transmission schemes with feedback are described in details, especially for the Type II Hybrid ARQ scheme. 
In Chapter 3, we focus on the rate allocation problem of the transmission scheme with feedback constraint on each packet. The problem is formulated first, and then the solutions based on rate-optimal and distortion-optimal approaches are proposed.

In Chapter 4, the simulation model and methodology are described first, and then simulation results of our proposed solutions are presented and compared with those of previous work.

In Chapter 5, we conclude the thesis and give a few suggestions for future work. 


\section{Chapter 2 Background and Literature Review}

\subsection{Image Transmission over Noisy Channels}

A general block diagram of image transmission over noisy channels is shown in Figure 2-1. At the transmitter, the original image is encoded by source encoder and channel encoder, and then transmitted over the noisy channel. Upon receiving the bits, the receiver reconstructs the image after channel decoding and source decoding and as long as the packets are all received correctly. We assume that there is an error detection mechanism available at the receiver which identifies the first erroneous packet and discards any packets received afterwards.

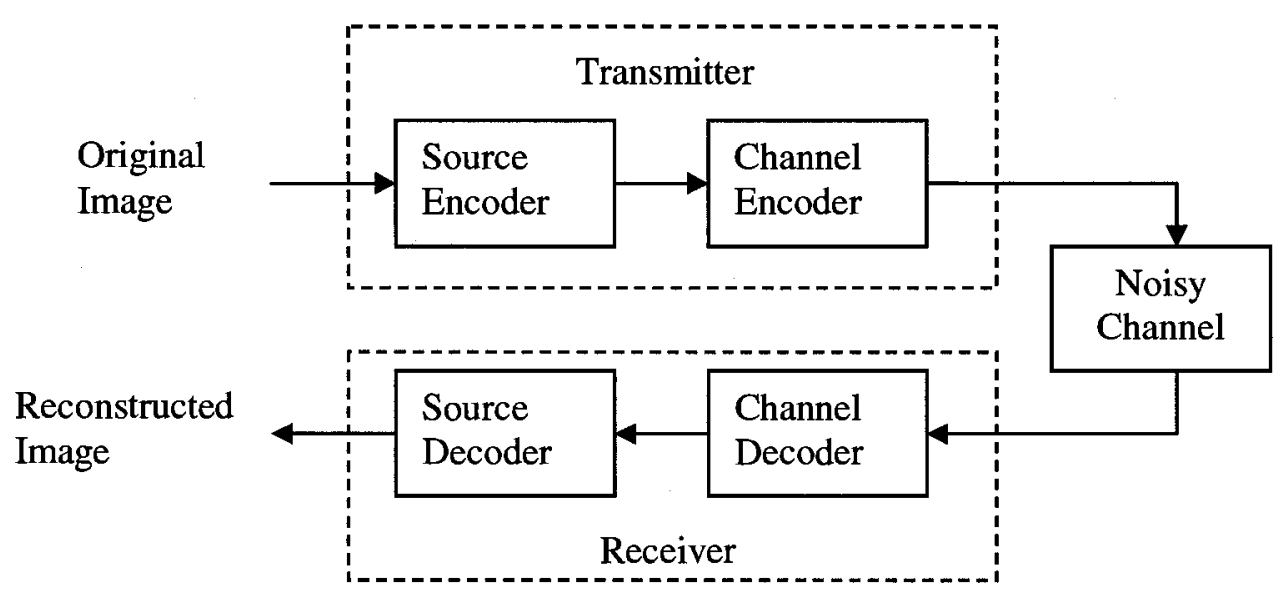

Figure 2-1 Block diagram of image transmission over a noisy channel

Due to the bandwidth constraint of the channel, image compression is necessary for reducing the number of bits to be transmitted. Source coding uses fewer bits to represent the original image by data compression techniques. Progressive source coding, which 
generates an output bitstream according to the importance of source bits, is particularly desirable for image transmission over noisy channels since it allows the receiver to reconstruct the image at different bit rates from the prefixes of the bitstream, providing a continually improving approximation to the original image. In the progressive image transmission, the outline of the original image can be identified with a small number of received bits although the quality is probably not very good. As more received bits are correctly decoded, the quality of the reconstructed image will be gradually improved. This can be seen from the example in Figure 2-2. Many source coders can generate progressive bitstreams for images. Among them, joint photographic experts group 2000 (JPEG2000) [1]-[2] and set partitioning in hierarchical trees (SPIHT) [3] are most widely used. The progressive bitstream is organized in a decreasing order of bits importance.
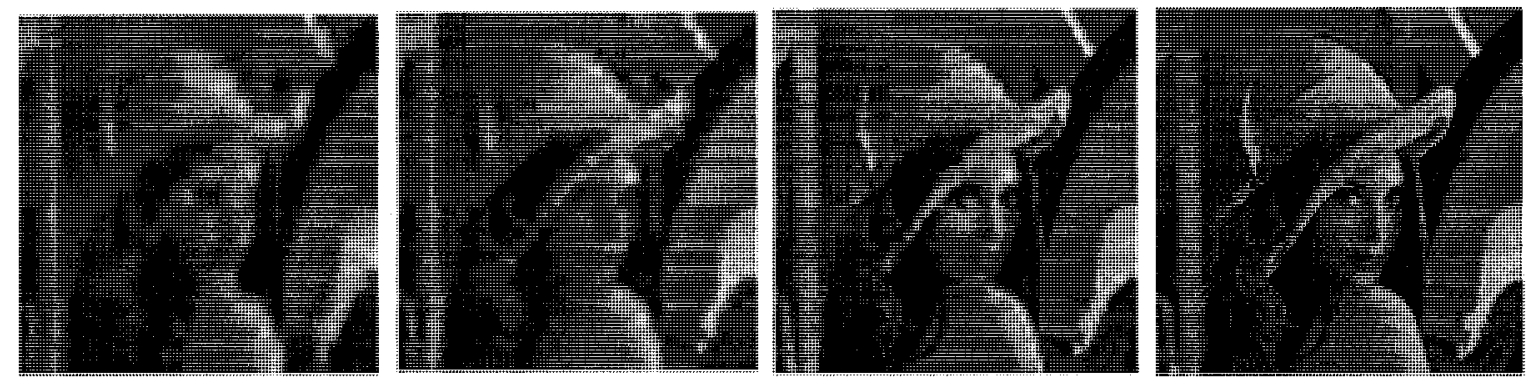

Figure 2-2 Progressive image transmission

However, a big drawback of progressive bitstreams is their high sensitivity to bit errors. A single bit error in the bitstream may cause error propagation to the later bits and a loss in synchronization between the encoder and the decoder. As a result, the bits after the error may degrade the quality of image reconstruction instead of improving the 
quality. Therefore, error control techniques are necessary to protect the bitstream against channel errors when the image transmission is on a noisy channel [4].

Forward error correction (FEC) and automatic repeat request (ARQ) are two available techniques for error control [5]-[6]. For a one-way noisy channel or for a strictly realtime transmission, only FEC can be used. In such situations, channel coding is employed for nearly error-free transmission of progressively coded images.

Traditionally, source coding and channel coding are independently considered and designed. However, it is well-known that joint source and channel coding (JSCC) can achieve a better end-to-end performance when a total transmission rate is given [7]. In a packet transmission system, a progressive bitstream is divided into packets before channel encoding. There are two kinds of channel coding for the packets: Equal error protection (EEP) and unequal error protection (UEP). EEP provides error protection to all packets with a same channel code rate regardless of the importance of the packets [8], while UEP provides error protection to each packet with a different channel code rate according to the importance of the packet [9]. It is shown that UEP outperforms EEP in joint source and channel coding [10]-[11]. A good way to implement UEP is to use ratecompatible ( $\mathrm{RC}$ ) codes, which have two attractive advantages: (1) the parity bits for higher rate codes are embedded in those of lower rate codes; (2) all codes can be encoded and decoded using a single encoder/decoder pair.

In recent years, researchers have used different kinds of $\mathrm{RC}$ codes to provide unequal error protection to the transmission of progressively coded images. RC punctured convolutional (RCPC) codes [12], RC turbo codes (RCTC), RC irregular repeat- 
accumulate (RC-IRA) codes, and RC low-density parity-check (RC-LDPC) codes are respectively used in [7]-[8], [13], [9] and [14].

\subsection{Rate Allocation Algorithm}

Rate allocation is a key component of joint source and channel coding [7]. If the distortion-rate function of the source encoder, the channel conditions, a finite family of rate-compatible channel codes, the cost function for performance measurement, and the total bit budget are given, then the algorithm that has the task of assigning different channel code rates to different source packets so as to achieve an optimal value for the cost function is called rate allocation algorithm. A block diagram of rate allocation algorithm is illustrated in Figure 2-3. Before the source bitstream of the original image is transmitted over the noisy channel, it is packetized into packets with different lengths and protected by channel codes with different rates. The packetized packet at the output of the source encoder is called source packet and the packet at the output of the channel encoder is called channel packet. The number of bits for source packet can be equivalently represented in a rate, bits per pixel, and the number of bits for channel packet can also be equivalently represented in coding rate. In a packet transmission, how many bits or what rates will be assigned to the source packet and the channel packet is decided by the rate allocation algorithm. For transmission schemes without feedback, the rate decision of the rate allocation algorithm depends on four conditions: the distortion-rate function of the source encoder, the channel BER, the total bit budget and the cost function of the 
performance measurement. Besides these four conditions, for transmission schemes with feedback, the rate decision of the rate allocation algorithm also depends on one additional condition, the number of feedback bits. In such schemes, the rate allocation algorithm will adaptively change the rates for source packets and channel packets according to the received bit of ACK/NACK sent by the receiver. The end-to-end performance is evaluated by comparing the reconstructed image with the original one, based on a cost function. Three well-known cost functions are average mean squared error (MSE) distortion, average peak signal-to-noise-ratio (PSNR), and average number of correctly received source bits [15]-[16]. Following [16], the optimal solutions for the first two cost functions are called distortion-based optimal or briefly distortion optimal, and the optimal solution for the last cost function is called rate-based optimal or briefly rate-optimal.

In image processing domain, the MSE between the original image and the reconstructed one is defined as:

$$
M S E=\frac{1}{N_{1} N_{2}} \sum_{n_{1}=0}^{N_{1}-1} \sum_{n_{2}=0}^{N_{2}-1}\left(x\left[n_{1}, n_{2}\right]-\hat{x}\left[n_{1}, n_{2}\right]\right)^{2}
$$

where $N_{1}$ and $N_{2}$ are the size of the image, and $x\left[n_{1}, n_{2}\right]$ and $\hat{x}\left[n_{1}, n_{2}\right]$ are respectively the pixel values of the two images at position $\left(n_{1}, n_{2}\right)$. And PSNR, which is used to represent the quality of reconstructed image, is defined as:

$$
\operatorname{PSNR}(d B)=20 \log \frac{2^{B}-1}{R M S E}
$$

where $B$ is the number of bits to represent each pixel, and RMSE is the root mean squared error between two images, i.e. $R M S E=\sqrt{M S E}$. 
There are also two approaches to packetize source bitstream and construct channel packets before image transmission. Corresponding to these, there are also two types of rate allocation problem: variable-length packet problem (VPP) and fixed-length packet problem (FPP). For VPP, the lengths of all source packets are the same, but the lengths of channel packets are different after channel coding with different code rates [14]-[15], [17]. For FPP, the lengths of channel packets are kept the same, but the lengths of source packets are different, depending on which channel code rates are used [9], [13], [16].

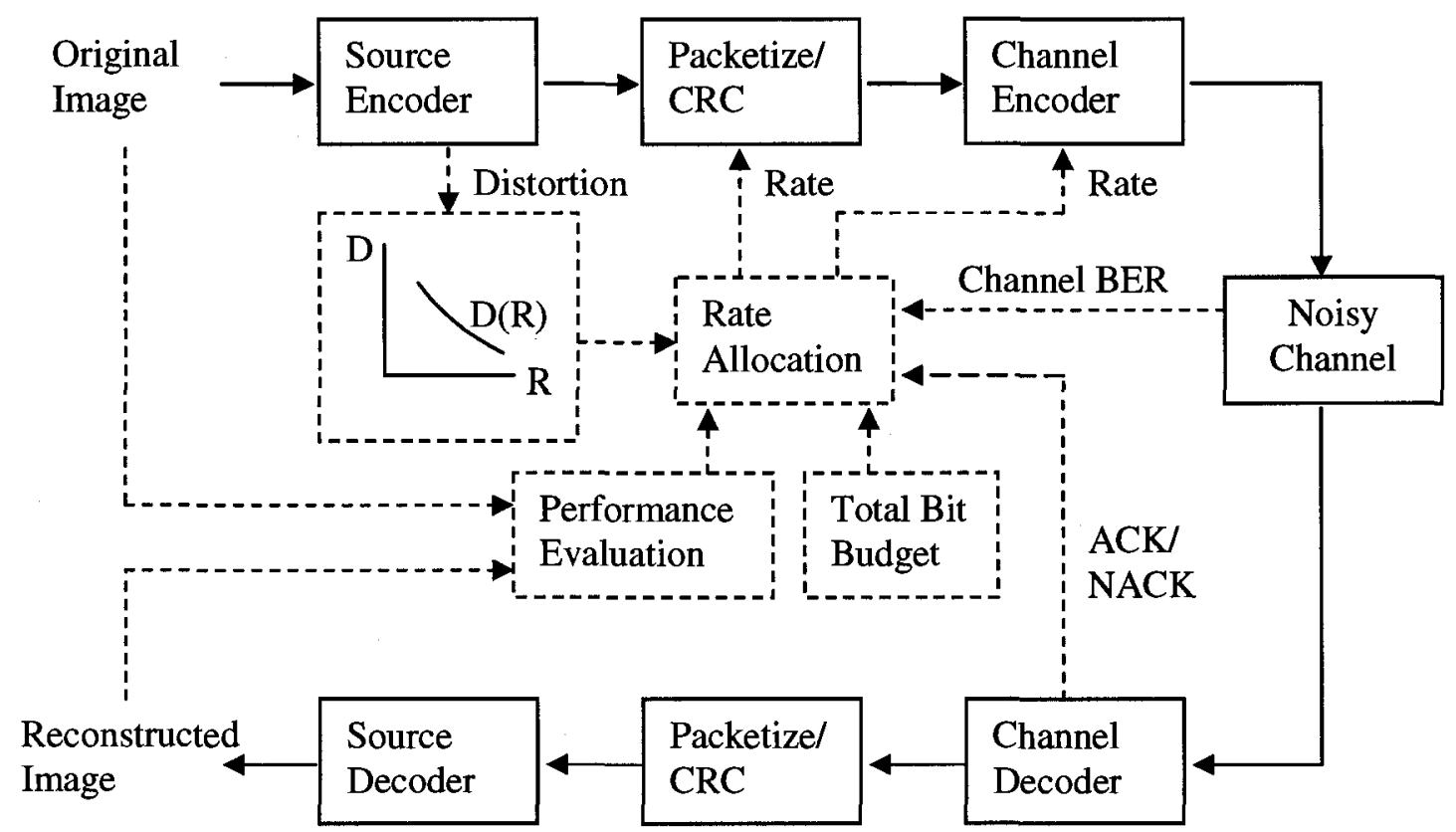

Figure 2-3 Block diagram of a transmission scheme with the rate allocation algorithm 


\subsection{Feedback Scheme}

If a feedback channel from the receiver to the transmitter is available and the delay is not of much concern, then ARQ scheme is often used for robust image transmission.

Hybrid ARQ (HARQ), a protocol which combines FEC and ARQ techniques, is more attractive since it exploits both the predictable performance of FEC codes and the rate flexibility of ARQ protocol. HARQ can achieve a better end-to-end performance than FEC and ARQ in image transmission over noisy channels if there is a constraint on total transmission bits [4]-[5], [12], [18]-[19].

A typical block diagram of image transmission over a noisy channel with HARQ is illustrated in Figure 2-4, where an error free feedback channel from the receiver to the transmitter is added to Figure 2-1, the block diagram of image transmission without ARQ.

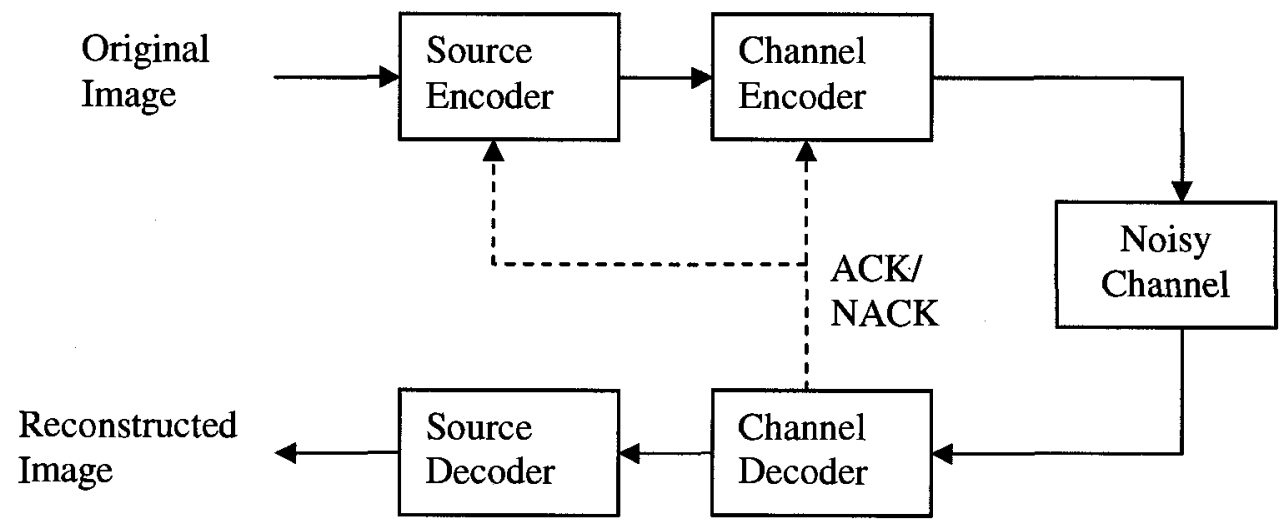

Figure 2-4 Block diagram of image transmission over noisy channel with HARQ 
There are two types of HARQ: Type I HARQ and Type II HARQ. The main difference between Type I HARQ and Type II HARQ is that Type I HARQ uses the same FEC code for retransmission attempts, while Type II HARQ uses different FEC codes for retransmission attempts. Type II HARQ is also called incremental redundancy HARQ. The incremental redundancy is usually achieved by using rate-compatible codes.

In this thesis, we will focus on Type II HARQ. In the following, we explain how this protocol works in a VPP transmission system.

Suppose that for a VPP transmission system, the total bit budget is given. The family of rate-compatible channel codes is $C=\left\{c_{1}, c_{2}, \ldots, c_{J}\right\}$ and the number of retransmission opportunities (or the number of available feedback bits) for each packet is $M-1$. A candidate policy to transmit a given packet with Type II HARQ can be written as

$$
\pi=\left(c_{\pi}^{1}, c_{\pi}^{2}, \ldots, c_{\pi}^{n(\pi)}\right)
$$

where $n(\pi) \leq M ; c_{\pi}^{1}, c_{\pi}^{2}, \ldots$, and $c_{\pi}^{n(\pi)}$ are chosen from the family of channel codes $C$ and their code rates are monotonically decreasing [20].

At the beginning of the transmission, the channel encoder uses the highest rate channel code $c_{\pi}^{1}$ to protect the source packet and transmits it over the noisy channel. At the receiver, the channel decoder attempts to decode the received bits and convey the decoding result (success or failure) to the transmitter by sending one bit (ACK/NACK) through the feedback channel, assuming the transmission of the feedback bit is error free. If a failure message NACK is received by the transmitter, the channel encoder will use the next lower rate channel code $c_{\pi}^{2}$ to protect the source packet, by only sending the 
incremental parity bits to the receiver. The channel decoder attempts to decode the packet again by appending the new received values to the previously received vector and conveys the decoding result to the transmitter. If a failure message NACK is continually received by the transmitter, the channel encoder will orderly use the lower rate channel codes $c_{\pi}^{3}, c_{\pi}^{4}, \ldots, c_{\pi}^{n(\pi)}$ to protect the source packet, by only sending the incremental parity bits to the receiver. When the lowest rate channel code $c_{\pi}^{n(\pi)}$ is used and a failure message is still received, the channel encoder and channel decoder will start another round of transmission for the packet if the feedback bits are still available. At the beginning of the new round, the channel encoder discards all previously transmitted bits and turn back to use code $c_{\pi}^{1}$ as error protection to retransmit the whole source packet. The channel decoder cleans the received bits stored in the decoding buffer and decodes the packet only depending on the newly received information. The procedure repeats as in the first round and may generate another new round, until the available feedback bits are exhausted or the total bit budget is exhausted or a success message is received by the transmitter. If a success message ACK is received, the channel encoder will stop the transmission of the current packet and proceeds with the transmission of the next packet. Once the available feedback bits for a given packet are exhausted and the packet is till in error or when the total bit budget is exhausted, the image transmission is terminated and the end-to-end performance is computed based on the correctly received packets at the receiver. 


\section{Chapter 3 Rate Allocation for Feedback Channels}

\section{with a Constraint on the Number of Feedback Bits per}

\section{Packet}

In this chapter, we will discuss the rate allocation problem of progressive image transmission over noisy channels with feedback constraint on each packet. Feedback constraint on each packet means that the number of feedback bits for each packet is fixed. We intend to find rate-optimal and distortion-optimal solutions for this problem. There are three sections in this chapter. The problem is formulated first, and then two solutions are proposed. Finally, the performances of the solutions are analyzed and compared.

\subsection{Problem Description}

Assume that a progressive image transmission over a noisy channel with feedback has the following conditions:

1. It is VPP packetized;

2. The total bit budget is given;

3. The transmission scheme is Type II HARQ;

4. A given family of rate-compatible channel codes is used;

5. The number of feedback bits for each packet is given.

The problem we are going to discuss and solve is how to choose a transmission policy for each packet so as to obtain an optimal (or suboptimal) end-to-end performance (in PSNR) 
before a packet decoding is failed or when the target bit budget is exhausted, under the above conditions.

Before the discussion of the proposed solutions, let us define some common symbols that are used throughout the rest of the thesis. Let $C=\left\{c_{1}, c_{2}, \ldots, c_{J}\right\}$ denote a family of rate-compatible channel codes such that the corresponding code rates are decreasing, $r_{c}\left(c_{i}\right)$ denote the code rate of $c_{i}, P_{e}\left(c_{i}\right)$ denote the error probability of $c_{i}, b_{s}$ denote the number of source bits on each packet, $M-1$ denote the number of feedback bits for each packet, and $\pi \stackrel{\text { def }}{=}\left(c_{\pi}^{1}, c_{\pi}^{2}, \ldots, c_{\pi}^{n(\pi)}\right)$ (where $n(\pi) \leq M ; c_{\pi}^{1}, c_{\pi}^{2}, \ldots$, and $c_{\pi}^{n(\pi)}$ are chosen from the family of channel codes $C$ and their code rates are monotonically decreasing) denote a candidate policy to transmit a packet.

\subsection{Solutions}

\subsubsection{Solution 1: Rate-optimal Solution Proposed by Chande et al}

In [21], Chande, Jafarkhani and Farvardin proposed a rate-optimal solution to the problem discussed in the previous section. Their idea came from the fact that for a given bit budget in VPP progressive image transmission, minimizing the average number of transmission bits per packet is equivalent to maximizing the average number of transmitted packets considering the fixed total bit budget, which, in turn, would be equivalent to maximizing the average PSNR. 
The procedure of solution 1 to obtain the decision policy for each packet is the following:

1. Consider all candidate policies $\pi=\left(c_{\pi}^{1}, c_{\pi}^{2}, \ldots, c_{\pi}^{n(\pi)}\right)$;

2. Impose a constraint that the packet decoding failure probability is less than a certain threshold $p_{e}$;

3. Require the maximum number of feedback bits per packet to be less than or equal to $M$-1, i.e. $\mathrm{n}(\pi) \leq \mathrm{M}$;

4. Compute the average number of transmission bits per packet for a candidate policy with an approximate equation

$$
R(\pi)=\sum_{k=1}^{n(\pi)} \frac{b_{s}}{r_{c}\left(c_{\pi}^{k}\right)}\left(P_{e}\left(c_{\pi}^{k-1}\right)-P_{e}\left(c_{\pi}^{k}\right)\right)+\frac{b_{s}}{r_{c}\left(c_{\pi}^{n(\pi)}\right)} P_{e}\left(c_{\pi}^{n(\pi)}\right)
$$

where $P_{e}\left(c_{\pi}^{0}\right) \stackrel{\text { def }}{=} 1$

5. Minimize $R(\pi)$ subject to $P_{e}\left(c_{\pi}^{n(\pi)}\right) \leq p_{e}$ and $n(\pi) \leq M$;

6. Choose the policy with the minimum $R(\pi)$ value as the decision policy to transmit each packet.

Dynamic programming [24], a method of optimization, is used to find the policy with the minimum $R(\pi)$ value. In the simulations performed in [21], the threshold value $p_{e}$ is set to be 0.01 . 


\subsubsection{Solution 2: Our Proposed Rate-optimal Solution}

This solution is based on the average number of correctly received bits cost function for the transmission scheme without feedback. We however make modifications to match this cost function to the problem under consideration. The details are described below.

For the VPP transmission scheme without feedback, the transmission policy for one packet is a single channel code chosen from the family of channel codes. Each packet has only one opportunity for transmission. Once a packet transmission is failed, the image transmission is terminated immediately and the end-to-end performance is computed based on the correctly received packets at the receiver: Suppose the image transmission policy for the VPP transmission scheme without feedback is $\Omega=\left(c_{\Omega}^{1}, c_{\Omega}^{2}, \ldots, c_{\Omega}^{t(\Omega)}\right)$, where $\mathrm{t}(\Omega)$ is the number of transmitted packets. This means we use channel code $c_{\Omega}^{1}$ to encode the first packet, use $c_{\Omega}^{2}$ to encode the second packet, use $c_{\Omega}^{i}$ to encode the $i$ th packet, and so on. Then the cost function for the rate-optimal solution can be represented by the equation

$$
V_{\Omega}=\sum_{i=1}^{t(\Omega)-1} b_{s} i \bullet P_{e}\left(c_{\Omega}^{i+1}\right) \bullet \prod_{j=1}^{i}\left(1-P_{e}\left(c_{\Omega}^{j}\right)\right)+b_{s} t(\Omega) \cdot \prod_{i=1}^{t(\Omega)}\left(1-P_{e}\left(c_{\Omega}^{i}\right)\right)
$$

where $i$ is the packet number, and $P_{e}\left(c_{\Omega}^{i}\right)$ is the packet error probability of the channel code $c_{\Omega}^{i}$ [15]. Meanwhile, for a given bit budget, we also need to count the total number of transmission bits that have been exhausted after each packet is transmitted. Once the target bit budget is reached, the image transmission is terminated. The equation to calculate the total number of transmission bits is 


$$
B_{\Omega}=\sum_{i=1}^{t(\Omega)} \frac{b_{s}}{r_{c}\left(c_{\Omega}^{i}\right)}
$$

where $r_{c}\left(c_{\Omega}^{i}\right)$ is the code rate of $c_{\Omega}^{i}$, and $\frac{b_{s}}{r_{c}\left(c_{\Omega}^{i}\right)}$ is the number of transmission bits of the $i$ th packet. Clearly, the error probability $P_{e}\left(c_{\Omega}^{i}\right)$ in Equation (3-2) and the number of transmission bits $\frac{b_{s}}{r_{c}\left(c_{\Omega}^{i}\right)}$ in Equation (3-3) are fully determined by a single channel code $c_{\Omega}^{i}$, a policy on one packet.

For the VPP transmission scheme with feedback, the transmission policy for one packet is a sequence of channel codes, not a single channel code. If the number of feedback bits on each packet is $M-1$, then a candidate policy can be written as $\pi=\left(c_{\pi}^{1}, c_{\pi}^{2}, \ldots, c_{\pi}^{n(\pi)}\right)$, where $n(\pi) \leq M$. All candidate policies compose a family of candidate policies

$$
\eta=\left\{\pi_{1}, \pi_{2}, \ldots, \pi_{Y}\right\}
$$

where $\pi_{\mathrm{i}} \stackrel{\text { def }}{=}\left(c_{\pi_{\mathrm{i}}}^{1}, c_{\pi_{\mathrm{i}}}^{2}, \ldots, c_{\pi_{\mathrm{i}}}^{n\left(\pi_{\mathrm{i}}\right)}\right), n\left(\pi_{\mathrm{i}}\right) \leq M$. Therefore, an image transmission policy can be represented as

$$
\Omega=\left(\pi_{\Omega}^{1}, \pi_{\Omega}^{2}, \ldots, \pi_{\Omega}^{t(\Omega)}\right)
$$

where $\pi_{\Omega}^{1}, \pi_{\Omega}^{2}, \ldots, \pi_{\Omega}^{t(\Omega)}$ are the transmission policies for the packet $1,2, \ldots$, and $t(\Omega)$, chosen from the family of candidate policies $\eta$, respectively. The key point for the transmission scheme with feedback to use Equations (3-2) and (3-3), originally used for 
the scheme without feedback, is to find the error probability and the number of transmission bits for each candidate policy. The error probability and the number of transmission bits should be equivalent to the parameters $P_{e}\left(c_{\Omega}^{i}\right)$ in Equation (3-2) and $\frac{b_{s}}{r_{c}\left(c_{\Omega}^{i}\right)}$ in Equation (3-3), respectively. To achieve this, we use the average number of transmission bits and the average error probability for the transmission policy for one packet.

For a packet transmission policy $\pi=\left(c_{\pi}^{1}, c_{\pi}^{2}, \ldots, c_{\pi}^{n(\pi)}\right)$, where $n(\pi) \leq M$, we compute the average number of transmission bits by using equation

$$
\begin{aligned}
& R(\pi)=\sum_{k=1}^{M-1}\left\{\left(\frac{b_{s}}{r_{c}\left(c_{\pi}^{n(\pi)}\right)} \times\left\lfloor\frac{k-1}{n(\pi)}\right\rfloor+\frac{b_{s}}{r_{c}\left(c_{\pi}^{\left.k \dashv \frac{k-1}{n(\pi)}\right\rfloor(\pi)}\right)}\right) \times\left(\prod_{i=1}^{n(\pi)} P_{e}\left(c_{\pi}^{i}\right)\right)^{\left\lfloor\frac{k-1}{n(\pi)}\right\rfloor}\right. \\
& \left.\times \prod_{j=0}^{k \dashv\left[\frac{k-1}{n(\pi)}\right\rfloor \times n(\pi)-1} P_{e}\left(c_{\pi}^{j}\right)\left(1-P_{e}\left(c_{\pi}^{k-\left\lfloor\frac{k-1}{n(\pi)}\right\rfloor \times n(\pi)}\right)\right)\right\} \\
& +\left(\frac{b_{s}}{r_{c}\left(c_{\pi}^{n(\pi)}\right)} \times\left\lfloor\frac{M-1}{n(\pi)}\right\rfloor+\frac{b_{s}}{r_{c}\left(c_{\pi}^{M-\left\lfloor\frac{M-1}{n(\pi)}\right\rfloor \times n(\pi)}\right)} \times\left(\prod_{i=1}^{n(\pi)} P_{e}\left(c_{\pi}^{i}\right)\right)^{\left\lfloor\frac{M-1}{n(\pi)}\right\rfloor}\right. \\
& M-\left\lfloor\frac{M-1}{n(\pi)}\right\rfloor \times n(\pi)-1 \\
& \times \prod_{j=0} P_{e}\left(c_{\pi}^{j}\right)
\end{aligned}
$$


where $P_{e}\left(c_{\pi}^{0}\right) \stackrel{\text { def }}{=} 1$ and $\left\lfloor\frac{x}{n(\pi)}\right\rfloor$ is the largest integer less than or equal to $\frac{x}{n(\pi)}$. We also compute the average error probability by using equation

$$
P_{e}(\pi)=\left(\prod_{i=1}^{n(\pi)} P_{e}\left(c_{\pi}^{i}\right)\right)^{\left\lfloor\frac{M}{n(\pi)}\right\rfloor} \bullet \prod_{j=0}^{M-\left\lfloor\frac{M}{n(\pi)} \mid \times n(\pi)\right.} P_{e}\left(c_{\pi}^{j}\right)
$$

where $P_{e}\left(c_{\pi}^{0}\right) \stackrel{\text { def }}{=} 1$ and $\left\lfloor\frac{x}{n(\pi)}\right\rfloor$ is the largest integer less than or equal to $\frac{x}{n(\pi)}$. In deriving (3-7), we have assumed that the failures of different codes in correcting the packet are independent.

By replacing $P_{e}\left(c_{\Omega}^{i}\right)$ in Equation (3-2) with the average error probability $P_{e}\left(\pi_{\Omega}^{i}\right)$ of the policy $\pi_{\Omega}^{i}$, we obtain the following cost function for the transmission scheme with feedback as:

$$
V_{\Omega}=\sum_{i=1}^{t(\Omega)-1} b_{s} i \bullet P_{e}\left(\pi_{\Omega}^{i+1}\right) \bullet \prod_{j=1}^{i}\left(1-P_{e}\left(\pi_{\Omega}^{j}\right)\right)+b_{s} t(\Omega) \bullet \prod_{i=1}^{t(\Omega)}\left(1-P_{e}\left(\pi_{\Omega}^{i}\right)\right)
$$

Similarly, by replacing $\frac{b_{s}}{r_{c}\left(c_{\Omega}^{i}\right)}$ in Equation (3-3) with the average number of transmitted bits $R\left(\pi_{\Omega}^{i}\right)$ of the policy $\pi_{\Omega}^{i}$, we obtain the total average number of transmitted bits for the transmission scheme with feedback as: 


$$
B_{\Omega}=\sum_{i=1}^{t(\Omega)} R\left(\pi_{\Omega}^{i}\right)
$$

Based on the cost function of (3-8) and by using Equation (3-9) for the constraint on the total bit budget, we can use either the forward Viterbi Algorithm [13] or backward dynamic programming algorithm [15], [22] to obtain the optimal policy for each packet in the transmission scheme with feedback. Note that in the case of feedback, a packet transmission policy is treated similar to a single channel code in the no feedback scenario.

Equation (3-9) is used to impose the constraint on the total bit budget in the optimization. Since this equation uses the average number of transmission bits of candidate policies to count the total number of transmission bits, it is possible that the number of polices obtained by Solution 2 is not enough for the practical image transmission. In other words, the actual number of packets that can be successfully transmitted in a practical image transmission may be larger than the expected number of packets that Solution 2 computes. In such cases, the additional packets will use the last policy of Solution 2 as their transmission policy. In our simulations, we notice that this special case does happen, but not often. When it happens, the number of additional packets is not more than 2 .

Since in general the number of candidate policies in the set $\eta$ is large, we have developed a number of techniques to reduce the computational complexity of Solution 2 . These are discussed in details in Section 3.3. 
The implementation procedure of Solution 2 can be summarized as follows.

1. Create the set $\eta$ of all possible policies $\pi$ which meet the constraint $\mathrm{n}(\pi) \leq M$;

2. Compute the average error probability for each policy by using Equation (37);

3. Compute the average number of transmission bits for each policy by using Equation (3-6);

4. Reduce the number of candidate policies by using measures described in Section 3.3;

5. Use either the forward Viterbi Algorithm [13] or backward dynamic programming algorithm [15], [22] to get the optimal policy for each packet based on the cost function of (3-8) and using (3-9) in the constraint $B_{\Omega} \leq B_{\text {tot }}$, where $B_{t o t}$ is the total bit budget.

We should notice that, if the number of feedback bits is zero, i.e. $\mathrm{n}(\pi)=M=1$, then $P_{e}\left(\pi_{\Omega}^{i}\right)$ is equal to $P_{e}\left(c_{\Omega}^{i}\right), R\left(\pi_{\Omega}^{i}\right)$ is equal to $\frac{b_{s}}{r_{c}\left(c_{\Omega}^{i}\right)}$, Equation (3-9) becomes Equation (3-3), and Equation (3-8) becomes Equation (3-2). This means Solution 2 is also applicable for the FEC scheme without feedback. In fact, the FEC scheme is a special case of the transmission scheme with feedback, where the number of feedback bits is zero. Therefore, we can use a single solution - Solution 2 to solve the rate-allocation problem for both the transmission scheme with feedback and the FEC scheme without feedback. 


\subsubsection{Solution 3: Proposed Distortion-optimal Solution}

This solution is based on using the average distortion as the cost function. Similar to Solution 2, we modify the formulas for the transmission scheme with no feedback to be applicable to the system with feedback. The only difference between Solution 3 and Solution 2 is that Solution 3 uses the distortion cost function to obtain the optimal policy for each packet, while Solution 2 uses the average number of correctly received bits as cost function.

Suppose that the image transmission policy for the VPP transmission scheme without feedback is $\Omega=\left(c_{\Omega}^{1}, c_{\Omega}^{2}, \ldots, c_{\Omega}^{t(\Omega)}\right)$, which means we use channel code $c_{\Omega}^{1}$ to encode the first packet, use $c_{\Omega}^{2}$ to encode the second packet, use $c_{\Omega}^{i}$ to encode the $i$ th packet, an so on. Then the average distortion cost function can be represented as

$$
D=D_{0} P_{e}\left(c_{\Omega}^{1}\right)+\sum_{j=1}^{t(\Omega)-1} D_{j} P_{e}\left(c_{\Omega}^{j+1}\right) \prod_{i=1}^{j}\left(1-P_{e}\left(c_{\Omega}^{i}\right)\right)+D_{t(\Omega)} \prod_{i=1}^{t(\Omega)}\left(1-P_{e}\left(c_{\Omega}^{i}\right)\right)
$$

where $D_{j}=D\left(b_{s} j\right), 1 \leq j \leq t(\Omega)$, is the distortion associated with the erroneous $(j+1)$ th packet (assuming all the previous packets have been correctly received), and $D_{0}$ is the source variance [22], [15].

Similar to Solution 2, by replacing $P_{e}\left(c_{\Omega}^{i}\right)$ in Equation (3-10) with the average error probability $P_{e}\left(\pi_{\Omega}^{i}\right)$ of the policy $\pi_{\Omega}^{i}$, we can get the distortion cost function (MSE based) for the transmission scheme with feedback as: 


$$
D_{\Omega}=D_{0} P_{e}\left(\pi_{\Omega}^{1}\right)+\sum_{j=1}^{t(\Omega)-1} D_{j} P_{e}\left(\pi_{\Omega}^{j+1}\right) \prod_{i=1}^{j}\left(1-P_{e}\left(\pi_{\Omega}^{i}\right)\right)+D_{t(\Omega)} \prod_{i=1}^{t(\Omega)}\left(1-P_{e}\left(\pi_{\Omega}^{i}\right)\right)
$$

assuming that the image transmission policy is $\Omega=\left(\pi_{\Omega}^{1}, \pi_{\Omega}^{2}, \ldots, \pi_{\Omega}^{t(\Omega)}\right)$.

By simply replacing the distortion value in Equation (3-11) with the PSNR value, we get the distortion cost function (PSNR based) for the transmission scheme with feedback as:

$$
S_{\Omega}=S_{0} P_{e}\left(\pi_{\Omega}^{1}\right)+\sum_{j=1}^{t(\Omega)-1} S_{j} P_{e}\left(\pi_{\Omega}^{j+1}\right) \prod_{i=1}^{j}\left(1-P_{e}\left(\pi_{\Omega}^{i}\right)\right)+S_{t(\Omega)} \prod_{i=1}^{t(\Omega)}\left(1-P_{e}\left(\pi_{\Omega}^{i}\right)\right)
$$

where $S_{j}$ is the PSNR value corresponding to the reconstruction of the image with $b_{s} j$ bits, $0 \leq j \leq t(\Omega)$.

Except the cost function and computational complexity, the implementation procedure of Solution 3 is completely the same as that of Solution 2.

\subsection{Measures to Reduce the Computational Complexity of Solutions 2}

\section{and 3}

In order to reduce the computational complexity of Solutions 2 and 3, three measures are used to reduce the number of candidate policies as well as the number of states at each level of the trellis when we use either forward Viterbi algorithm or backward dynamic programming approach to get the optimal transmission policy for each packet. 
1. For two possible policies $\pi_{1}$ and $\pi_{2}$, if the average number of transmission bits of $\pi_{2}$ is greater than that of $\pi_{1}$, but the average error probability of $\pi_{2}$ is not smaller than that of $\pi_{1}$, then $\pi_{2}$ will be eliminated from the set of candidate policies and $\pi_{1}$ will remain. If $\pi_{1}$ and $\pi_{2}$ have the same average error probabilities but different average number of transmission bits, then the policy with a greater average number of transmission bits will be eliminated and the other will remain. This measure can reduce the number of possible policies.

2. When the average error probability of a policy is less than $1 \times 10^{-5}$, it is assumed to be zero. There are three reasons: (1). From the practical point of view, error probabilities smaller than $1 \times 10^{-5}$ have minimal effect on the cost function and are equivalent to zero. (2). Our experiments show that we can achieve a better end-to-end performance with round threshold value $1 \times 10^{-5}$ than with $2 \times 10^{-5}$, or $1 \times 10^{-4}$, or $1 \times 10^{-6}$. (3). The computational complexity of the proposed solutions after using the round threshold value $1 \times 10^{-5}$ is moderate and acceptable. By this measure, the number of possible policies will be greatly reduced especially for large number of feedback bits. From Equation (3-7), we know the average error probability of a policy is the product of error probabilities of the channel codes in the policy. So for large number of feedback bits, more policies will have an average error probability of 0 . Only one of these policies can then remain as a candidate policy according to Measure 1 . 
3. The average number of transmission bits of a policy obtained by Equation (36) is not necessarily an integer number. We round the average number of transmission bits to the nearest integer. By doing so, when we use either forward Viterbi algorithm or backward dynamic programming approach to get the optimal transmission policy for each packet, the number of states at each level of the trellis will not increase exponentially as the number of packets increases. Since the average number of transmission bits is rounded to an integer, many combinations of policies arriving at certain level of the trellis will have the same accumulated number of transmission bits and will be assigned to the same state. Therefore, by applying the Viterbi algorithm or the backward dynamic programming, only the policy with the largest useful source bits or the smallest distortion value can survive for this state. The complexity [number of calls to the recursive function in forward VA or backward dynamic programming algorithm to achieve the optimal policy] of Solution 2 is $O(R)$ and the complexity of Solution 3 is $O\left(R^{2}\right)$, where $R$ is the total bit budget. For the discussion on complexity, please refer to [15]. In fact, a state at a certain level of the trellis is associated with a certain number of accumulative transmission bits. Such number of accumulative transmission bits can not exceed the total bit budget, and the number of accumulative transmission bits is an integer, so the number of states at a level will never be larger than the number of total bit budget. For example, if the total bit budget $=1.0 \mathrm{bpp}$, then the number of states at a level will not be larger than $1.0 \times 512 \times 512 / 8=32,768$. In fact in our 
simulations, the number of states at a level is much less than 32,768 after using the above three simplification measures.

\subsection{Comparison of the Proposed Solutions with Solution 1}

Before evaluating and comparing the performance of the three solutions by simulations, we discuss a few differences that exist among the solutions.

Solutions 1 and 2 both use the number of correctly received bits as the cost function, while Solution 3 use the distortion as the measure of performance.

Solution 2 is superior to Solution 1 because:

1. Solution 2 uses a precise equation to compute the average number of transmission bits for a policy, while Solution 1 uses an approximate equation.

2. Solution 2 does not need to specify a threshold value $p_{e}$ for the error probability of a candidate policy, but Solution 1 has to do so. How to specify an appropriate $p_{e}$ for different scenarios is an important issue which has not been discussed in [21].

3. By using Solution 2, the transmission policy may vary from packet to packet, but by using Solution 1, a single policy is used for all packets. This is as if EEP is used for Solution 1 while Solution 2 uses UEP.

4. The performance of Solution 2 can be predicted, while the performance of Solution 1 can not be predicted. For Solution 2, we can get the average number of transmission bits and the average error probability for the policy on each packet by Equation (3-6) and Equation (3-7), respectively. Then we can use 
Equation (3-8) to obtain the average number of source bits under the constraint of Equation (3-9). Like the FEC scheme, the performance of Solution 2 can be obtained by calculation, without simulations. Our experiments show that the performances of Solution 2 by using equation and by simulation are very close. However, Solution 1 only provides a policy on each packet. It doesn't tell us how to compute the average number of transmission bits and the average error probability for the policy on each packet, and therefore can not use the cost function to predict the performance.

5. Solution 3 slightly outperforms Solution 2 in most cases and has a substantially better performance than Solution 1. The computational complexity of Solution 3 is however higher than both Solutions 1 and 2 . The improvement of Solution 3 over Solution 2 is usually larger when the average PSNR is used as the cost function.

\subsection{Upper and Lower Bounds on Performance}

The rate allocation solution to the ARQ scheme with feedback constraint on each packet should satisfy the following necessary criteria.

1. The performance of the proposed solution should be better than that of the FEC scheme, which has no feedback. We can regard the performance of the FEC scheme as the lower bound of the performances of ARQ schemes. 
2. As the number of feedback bits for each packet increases, the performance of the proposed solutions should continue to enhance and eventually converge to the performance of ARQ scheme with infinite number of feedback bits. For ARQ scheme with infinite number of feedback bits, each packet is re-transmitted without any limitation on the number of feedback bits until the transmission of the packet is successful or the total bit budget is exhausted. In such a scheme, the transmission policy for each packet is the entire family of rate-compatible channel codes ordered from the highest rate to the lowest rate, i.e. $\pi=\left(c_{1}, c_{2}, \ldots, c_{J}\right)$. The performance of ARQ scheme with infinite number of feedback bits is better than any ARQ scheme with finite number of feedback bits. We can regard the performance of ARQ scheme with infinite number of feedback bits as the upper bound of the performances of the proposed ARQ schemes.

The above criteria are very important to evaluate the performance of the proposed schemes as the gap between the bounds and the performance of the proposed scheme provides a good measure of the quality of the scheme. 


\section{Chapter 4 Simulation Results}

\subsection{Simulation Model and Methodology}

The simulations are conducted using the standard test image Lena, of gray scale, 8 bits per pixel (bpp), and with dimensions $512 \times 512$. The source image is compressed with JPEG2000 by Kakadu software [28] first, and then packetized into fix-length packets of 384 bytes. Each packet is transmitted with Type II HARQ scheme, protected by a sequence of channel codes.

For the Type II HARQ transmission scheme as we discussed in Section 2.3, each packet has a policy $\pi=\left(c_{\pi}^{1}, c_{\pi}^{2}, \ldots, c_{\pi}^{n(\pi)}\right)$ for transmission. $c_{\pi}^{1}$ is the initial channel code for error protection of the packet transmission, while $c_{\pi}^{2}, c_{\pi}^{3}, \ldots, c_{\pi}^{n(\pi)}, c_{\pi}^{1}, \ldots$, are the sequence of channel codes for error protection if the packet transmission encoded by the previous channel codes fails. In the simulations, when a channel code $c_{\pi}^{i}$ is chosen from the policy to protect the current packet, we generate a random variable $X$ uniformly distributed in the interval $[0,1]$ to represent the transmission result of the packet. If $X$ is smaller than or equal to the error probability $P_{e}\left(c_{\pi}^{i}\right)$ of the channel code $c_{\pi}^{i}$, then we assume that the packet transmission with the channel code $c_{\pi}^{i}$ has failed. Otherwise, we assume that the packet transmission is successful. If the packet transmission fails and the available number of feedback bits is greater than zero and the total bit budget is not exhausted, then we choose code $c_{\pi}^{i+1}$ to correct the packet. Again we generate a random 
variable $X$ uniformly distributed in the interval $[0,1]$, compare it to the error probability $P_{e}\left(c_{\pi}^{i+1}\right)$ of the code $c_{\pi}^{i+1}$, and make a decision on whether the packet transmission fails or is successful by comparing the values of $X$ and $P_{e}\left(c_{\pi}^{i+1}\right)$. This procedure will continue using codes with the next lower rates until the packet transmission is successful, or the total bit budget is exhausted, or the packet transmission fails and the available number of feedback bits is zero. If the transmission of current packet is successful, and the total bit budget is not exhausted, then the transmission of next packet will start. At any point, if the total bit budget is exhausted or all the feedback bits for the transmission of a packet are exhausted but the packet is still in error, the transmission is stopped. After the transmission is terminated, the image is reconstructed at the receiver with the successfully transmitted source bits by Kakadu software. Then an end-to-end PSNR value based on the difference between the original image and the reconstructed one will be calculated by the Jasper software implementation of JPEG2000 standard [29]. The final average PSNR value for each scenario is obtained by averaging over 50,000 trials.

Instead of Monte Carlo simulations, one can also use Equation (3-12) to obtain the average PSNR for different scenarios. Our experiments show that the difference between the two methods, although larger for smaller total bit budgets, is negligible.

In our simulations, the family of channel codes is rate-compatible LDPC codes, and the test channel is the correlated Rayleigh fading channel with SI. We use the same codes designed in [23]. Similar to [23], three different vehicle speeds $(6 \mathrm{~km} / \mathrm{h}, 50 \mathrm{~km} / \mathrm{h}$ and 120 $\mathrm{km} / \mathrm{h}$ ) are considered. For each vehicle speed, the performance of the rate-compatible 
LDPC codes in the correlated Rayleigh fading channels is obtained from [23]. This is described in the following subsection.

In order to help evaluate the performance of the proposed solutions and make comparisons, we also perform simulations for the ARQ scheme with infinite number of feedback bits and also for the FEC scheme.

For the ARQ scheme with infinite number of feedback bits, each packet uses the whole set of rate-compatible channel codes as the transmission policy, i.e. $\pi=C=\left(c_{1}, c_{2}, \ldots, c_{J}\right) . c_{1}$ is the initial channel code for error protection of the packet transmission, while $c_{2}, c_{3}, \ldots, c_{J}, c_{1}, \ldots$, are the sequence of channel codes for error protection if the packet transmission encoded by the previous channel codes fails. The transmission of one packet can continue until the transmission is successful or the total bit budget is exhausted, without the limitation on the number of feedback bits. When the total bit budget is exhausted, the image transmission is terminated. We use the same simulation methodology described earlier in this section to get the average number of useful source bits over 50,000 trials, and then to reconstruct the image with the average number of useful source bits.

We can also get the number of useful source bits for the ARQ scheme with infinite number of feedback bits by throughput equation. For Type II hybrid ARQ scheme with packet transmission policy $\pi=\left(c_{\pi}^{1}, c_{\pi}^{2}, c_{\pi}^{3}, \ldots\right)$, the throughput $(\beta)$ is defined as:

$$
\beta=\frac{b_{s}}{\frac{b_{s}}{r_{c}\left(c_{\pi}^{1}\right)}\left(1-P_{e}\left(c_{\pi}^{1}\right)\right)+\sum_{i=2}^{+\infty} \frac{b_{s}}{r_{c}\left(c_{\pi}^{i}\right)}\left(1-P_{e}\left(c_{\pi}^{i}\right)\right) \prod_{j=0}^{i-1} P_{e}\left(c_{\pi}^{j}\right)}
$$


From the equation, we can see that the throughput is in fact the ratio between the source bits and total transmission bits. Therefore, we can obtain the number of useful source bits $S$ with the throughput $\beta$ and total bit budget $B_{\text {tot }}$ by the equation

$$
S=\beta \times B_{t o t} .
$$

Our experiments show that the number of useful source bits obtained by simulations and by equation are very close.

The performance of the FEC scheme is also based on the rate-optimal. The transmission policy on each packet is obtained by the rate-optimal cost function (3-2). As discussed in Section 3.2.2, the FEC scheme is a special case of ARQ scheme, where the number of feedback bits is zero. In our simulations, we use the same programming code as the Type II hybrid ARQ scheme to obtain the performance for the FEC scheme, just modifying the number of feedback bits to zero for the FEC scheme. 


\subsubsection{Correlated Rayleigh Fading Channel with SI: Vehicle Speed V=6 km/h}

For vehicle speed $\mathrm{V}=6 \mathrm{~km} / \mathrm{h}$, the set of code rates is $[8 / 10,8 / 11,8 / 12,8 / 13,8 / 15$, $8 / 16,8 / 18,8 / 20,8 / 22\}$, which corresponds to the set of codeword lengths $\{480,528,576$, $624,720,768,864,960,1056\}$. The number of information bits for each packet is fixed at 384 bytes. The error probabilities of the channel codes at different SNR values are obtained from Fig. 3(a) of [23] and shown in Table 4-1.

\begin{tabular}{|c|c|c|c|c|c|c|c|}
\hline $\begin{array}{c}\text { Code } \\
\text { Rates }\end{array}$ & $\mathbf{1 0}$ & $\mathbf{1 2}$ & $\mathbf{1 3}$ & $\mathbf{1 4}$ & $\mathbf{1 6}$ & $\mathbf{1 8}$ & $\mathbf{2 0}$ \\
\hline $\mathbf{8} / 10$ & $4.0 \times 10^{-1}$ & $3.0 \times 10^{-1}$ & $2.5 \times 10^{-1}$ & $2.1 \times 10^{-1}$ & $1.6 \times 10^{-1}$ & $9.0 \times 10^{-2}$ & $5.6 \times 10^{-2}$ \\
\hline $\mathbf{8} / 11$ & $3.0 \times 10^{-1}$ & $2.2 \times 10^{-1}$ & $1.8 \times 10^{-1}$ & $1.6 \times 10^{-1}$ & $1.0 \times 10^{-1}$ & $6.0 \times 10^{-2}$ & $3.0 \times 10^{-2}$ \\
\hline $\mathbf{8} / 12$ & $2.5 \times 10^{-1}$ & $1.8 \times 10^{-1}$ & $1.4 \times 10^{-1}$ & $1.0 \times 10^{-1}$ & $6.0 \times 10^{-2}$ & $3.0 \times 10^{-2}$ & $1.5 \times 10^{-2}$ \\
\hline $\mathbf{8} / 13$ & $2.0 \times 10^{-1}$ & $1.5 \times 10^{-1}$ & $1.0 \times 10^{-1}$ & $7.0 \times 10^{-2}$ & $3.5 \times 10^{-2}$ & $2.0 \times 10^{-2}$ & $8.0 \times 10^{-3}$ \\
\hline $\mathbf{8} / 15$ & $1.5 \times 10^{-1}$ & $8.0 \times 10^{-2}$ & $5.0 \times 10^{-2}$ & $4.0 \times 10^{-2}$ & $2.0 \times 10^{-2}$ & $9.0 \times 10^{-3}$ & $4.0 \times 10^{-3}$ \\
\hline $\mathbf{8} / 16$ & $1.0 \times 10^{-1}$ & $5.0 \times 10^{-2}$ & $3.0 \times 10^{-2}$ & $2.0 \times 10^{-2}$ & $1.0 \times 10^{-2}$ & $4.8 \times 10^{-3}$ & $1.7 \times 10^{-3}$ \\
\hline $\mathbf{8} / 18$ & $7.0 \times 10^{-2}$ & $3.0 \times 10^{-2}$ & $2.0 \times 10^{-2}$ & $1.3 \times 10^{-2}$ & $5.0 \times 10^{-3}$ & $2.0 \times 10^{-3}$ & $6.0 \times 10^{-4}$ \\
\hline $\mathbf{8} / \mathbf{2 0}$ & $4.0 \times 10^{-2}$ & $1.6 \times 10^{-2}$ & $8.5 \times 10^{-3}$ & $5.0 \times 10^{-3}$ & $1.7 \times 10^{-3}$ & $6.0 \times 10^{-4}$ & $2.0 \times 10^{-4}$ \\
\hline $\mathbf{8} / \mathbf{2 2}$ & $3.0 \times 10^{-2}$ & $1.0 \times 10^{-2}$ & $5.0 \times 10^{-3}$ & $2.5 \times 10^{-3}$ & $7.0 \times 10^{-4}$ & $2.0 \times 10^{-4}$ & 0 \\
\hline
\end{tabular}

Table 4-1 Packet error probabilities of channel codes for correlated Rayleigh fading channel with $\mathrm{SI}, \mathrm{V}=6 \mathrm{~km} / \mathrm{h}$ 


\subsubsection{Correlated Rayleigh Fading Channel with SI: Vehicle Speed V=50 km/h}

For vehicle speed $V=50 \mathrm{~km} / \mathrm{h}$, the set of code rates is $\{8 / 10,8 / 11,8 / 12,8 / 13,8 / 15$, $8 / 16,8 / 18,8 / 20,8 / 22\}$, which corresponds to the set of codeword lengths $[480,528,576$, $624,720,768,864,960,1056\}$.The number of information bits for each packet is fixed at 384 bytes. The error probabilities of the channel codes at different SNR values are obtained from Fig. 3(b) of [23] and shown in Table 4-2.

\begin{tabular}{|c|c|c|c|c|c|c|c|}
\hline $\begin{array}{c}\text { Code } \\
\text { Rates }\end{array}$ & 10 & 12 & 13 & 14 & 16 & 18 & 20 \\
\hline $\mathbf{8} / 10$ & $2.5 \times 10^{-1}$ & $2.0 \times 10^{-1}$ & $1.6 \times 10^{-1}$ & $1.2 \times 10^{-1}$ & $6.7 \times 10^{-2}$ & $3.5 \times 10^{-2}$ & $2.0 \times 10^{-2}$ \\
\hline $\mathbf{8 / 1 1}$ & $2.0 \times 10^{-1}$ & $1.5 \times 10^{-1}$ & $9.0 \times 10^{-2}$ & $6.0 \times 10^{-2}$ & $3.0 \times 10^{-2}$ & $1.3 \times 10^{-2}$ & $5.0 \times 10^{-3}$ \\
\hline $\mathbf{8 / 1 2}$ & $1.6 \times 10^{-1}$ & $7.0 \times 10^{-2}$ & $5.0 \times 10^{-2}$ & $3.0 \times 10^{-2}$ & $1.4 \times 10^{-2}$ & $5.0 \times 10^{-3}$ & $2.0 \times 10^{-3}$ \\
\hline $\mathbf{8} / 13$ & $1.2 \times 10^{-1}$ & $5.0 \times 10^{-2}$ & $3.2 \times 10^{-2}$ & $2.0 \times 10^{-2}$ & $7.0 \times 10^{-3}$ & $2.0 \times 10^{-3}$ & $7.0 \times 10^{-4}$ \\
\hline $\mathbf{8 / 1 5}$ & $7.0 \times 10^{-2}$ & $3.0 \times 10^{-2}$ & $2.0 \times 10^{-2}$ & $1.0 \times 10^{-2}$ & $3.3 \times 10^{-3}$ & $8.0 \times 10^{-4}$ & $2.0 \times 10^{-4}$ \\
\hline $\mathbf{8 / 1 6}$ & $4.0 \times 10^{-2}$ & $1.7 \times 10^{-2}$ & $9.0 \times 10^{-3}$ & $5.0 \times 10^{-3}$ & $1.3 \times 10^{-3}$ & $3.0 \times 10^{-4}$ & 0 \\
\hline $\mathbf{8} / 18$ & $2.0 \times 10^{-2}$ & $7.0 \times 10^{-3}$ & $4.0 \times 10^{-3}$ & $2.0 \times 10^{-3}$ & $4.6 \times 10^{-4}$ & $1.0 \times 10^{-4}$ & 0 \\
\hline $\mathbf{8 / 2 0}$ & $1.1 \times 10^{-2}$ & $2.8 \times 10^{-3}$ & $1.1 \times 10^{-3}$ & $5.0 \times 10^{-4}$ & $1.0 \times 10^{-4}$ & 0 & 0 \\
\hline $\mathbf{8} / \mathbf{2 2}$ & $5.5 \times 10^{-3}$ & $1.2 \times 10^{-3}$ & $5.0 \times 10^{-4}$ & $2.0 \times 10^{-4}$ & 0 & 0 & 0 \\
\hline
\end{tabular}

Table 4-2 Packet error probabilities of channel codes for correlated Rayleigh fading channel with $\mathrm{SI}, \mathrm{V}=50 \mathrm{~km} / \mathrm{h}$ 


\subsubsection{Correlated Rayleigh Fading Channel with SI: Vehicle Speed V=120 km/h}

For vehicle speed $V=120 \mathrm{~km} / \mathrm{h}$, the set of code rates is $\{8 / 10,8 / 10.5,8 / 11,8 / 12,8 / 13$, $8 / 15,8 / 16,8 / 18,8 / 20,8 / 22\}$, which corresponds to the set of codeword lengths $\{480$, $504,528,576,624,720,768,864,960,1056\}$.The number of information bits for each packet is fixed at 384 bytes. The error probabilities of the channel codes at different SNR values are obtained from Fig. 3(c) of [23] and shown in Table 4-3.

\begin{tabular}{|c|c|c|c|c|c|c|c|}
\hline $\begin{array}{c}\text { Code } \\
\text { rates }\end{array}$ & $\mathbf{1 0}$ & $\mathbf{1 2}$ & $\mathbf{1 3}$ & $\mathbf{1 4}$ & $\mathbf{1 6}$ & $\mathbf{1 8}$ & $\mathbf{2 0}$ \\
\hline $\mathbf{8} / \mathbf{1 0}$ & $2.4 \times 10^{-1}$ & $1.7 \times 10^{-1}$ & $1.1 \times 10^{-1}$ & $8.0 \times 10^{-2}$ & $4.5 \times 10^{-2}$ & $2.0 \times 10^{-2}$ & $8.0 \times 10^{-3}$ \\
\hline $\mathbf{8 / 1 0 . 5}$ & $1.8 \times 10^{-1}$ & $8.0 \times 10^{-2}$ & $6.0 \times 10^{-2}$ & $3.3 \times 10^{-2}$ & $1.5 \times 10^{-2}$ & $6.5 \times 10^{-3}$ & $2.8 \times 10^{-3}$ \\
\hline $\mathbf{8 / 1 1}$ & $8.0 \times 10^{-2}$ & $4.2 \times 10^{-2}$ & $3.0 \times 10^{-2}$ & $1.6 \times 10^{-2}$ & $7.0 \times 10^{-3}$ & $2.8 \times 10^{-3}$ & $8.2 \times 10^{-4}$ \\
\hline $\mathbf{8 / 1 2}$ & $6.0 \times 10^{-2}$ & $2.5 \times 10^{-2}$ & $1.5 \times 10^{-2}$ & $8.0 \times 10^{-3}$ & $2.8 \times 10^{-3}$ & $8.0 \times 10^{-4}$ & $2.0 \times 10^{-4}$ \\
\hline $\mathbf{8 / 1 3}$ & $4.0 \times 10^{-2}$ & $1.5 \times 10^{-2}$ & $8.0 \times 10^{-3}$ & $4.5 \times 10^{-3}$ & $1.4 \times 10^{-3}$ & $3.0 \times 10^{-4}$ & 0 \\
\hline $\mathbf{8 / 1 5}$ & $2.5 \times 10^{-2}$ & $8.0 \times 10^{-3}$ & $5.0 \times 10^{-3}$ & $2.8 \times 10^{-3}$ & $5.0 \times 10^{-4}$ & $1.0 \times 10^{-4}$ & 0 \\
\hline $\mathbf{8 / 1 6}$ & $1.8 \times 10^{-2}$ & $5.0 \times 10^{-3}$ & $2.8 \times 10^{-3}$ & $1.4 \times 10^{-3}$ & $2.0 \times 10^{-4}$ & 0 & 0 \\
\hline $\mathbf{8 / 1 8}$ & $9.0 \times 10^{-3}$ & $2.5 \times 10^{-3}$ & $1.3 \times 10^{-3}$ & $5.0 \times 10^{-4}$ & 0 & 0 & 0 \\
\hline $\mathbf{8} / 20$ & $4.8 \times 10^{-3}$ & $9.0 \times 10^{-4}$ & $3.0 \times 10^{-4}$ & $1.0 \times 10^{-4}$ & 0 & 0 & 0 \\
\hline $\mathbf{8} / 22$ & $1.8 \times 10^{-3}$ & $3.0 \times 10^{-4}$ & $1.0 \times 10^{-4}$ & 0 & 0 & 0 & 0 \\
\hline
\end{tabular}

Table 4-3 Packet error probabilities of channel codes for correlated Rayleigh fading channel with SI, V=120 km/h 


\subsection{The Number of Candidate Policies Left in $\eta$ after Using the Simplification Measures}

In Section 3.3, three simplification measures are proposed to reduce the computational complexity for Solutions 2 and 3. The numbers of candidate policies left in $\eta$ for different cases after using the simplification measures are illustrated in Table 4-4. By comparison with the large number of candidate policies in $\eta$ before using the simplification measures, we can see that the number of candidate policies left in $\eta$ after using the simplification measures is very small, and it will decrease as the number of feedback bits increases, even reduces to 1 for many cases. This fact demonstrates that the three simplification measures are effective and efficient in reducing the computational complexity of Solutions 2 and 3. 


\begin{tabular}{|c|c|c|c|c|c|c|c|c|c|}
\hline \multirow{2}{*}{$\begin{array}{c}\text { Vehicle } \\
\text { Speed } \\
(\mathrm{km} / \mathrm{h})\end{array}$} & \multirow{2}{*}{$\begin{array}{c}\text { Number } \\
\text { of } \\
\text { Feedback } \\
\text { Bits }\end{array}$} & \multirow{2}{*}{ Before } & \multicolumn{7}{|c|}{ After } \\
\hline & & & $\begin{array}{c}S N R=10 \\
d B\end{array}$ & $\begin{array}{c}\text { SNR }=12 \\
\mathrm{~dB}\end{array}$ & $\begin{array}{c}S N R=13 \\
d B\end{array}$ & $\begin{array}{c}\text { SNR }=14 \\
d B\end{array}$ & $\begin{array}{c}\text { SNR }=16 \\
d B\end{array}$ & $\begin{array}{c}\text { SNR }=18 \\
d B\end{array}$ & $\begin{array}{c}\text { SNR }=20 \\
d B\end{array}$ \\
\hline \multirow{3}{*}{6} & 1 & 45 & 24 & 21 & 19 & 17 & 14 & 10 & 8 \\
\hline & 2 & 129 & 29 & 21 & 17 & 13 & 8 & 3 & 1 \\
\hline & 3 & $255 /$ & 28 & 13 & 7 & 2 & 2 & 1 & 1 \\
\hline & & & 18 & $16=$ & 14 & $-10^{-}$ & & 6 & 4 \\
\hline 350 & $\frac{(27)}{(3)}$ & $\frac{6}{2}-129$ & $\begin{array}{r}16 \\
-8 \\
\end{array}$ & $\sqrt{-2}$ & 2 & $\frac{-2}{-4}$ & $\frac{-2}{2}$ & $\frac{-1}{8}$ & 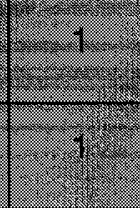 \\
\hline \multirow{3}{*}{120} & 1 & 55 & 18 & 14 & 13 & 9 & 6 & 4 & 1 \\
\hline & 2 & 175 & 14 & 8 & 3 & 1 & 1 & 1 & 1 \\
\hline & 3 & 385 & 3 & 1 & 1 & 1 & 1 & 1 & 1 \\
\hline
\end{tabular}

Table 4-4 The number of candidate policies in $\eta$ before and after using the simplification measures of Section 3.3

\subsection{Simulation Results of Solution 2}

In this section, we evaluate the performance of Solution 2 by simulations. We use Solution 2 to obtain the transmission policies for all packets first, and then use the simulation method described in Section 4.1 to perform simulations. The test channels are 
correlated Rayleigh fading channels with vehicle speed $V=6 \mathrm{~km} / \mathrm{h}, 50 \mathrm{~km} / \mathrm{h}$ and 120

$\mathrm{km} / \mathrm{h}$. For each vehicle speed, simulations are performed with $\mathrm{SNR}=10,14$ and $20 \mathrm{~dB}$, and the number of feedback bits=1, 2 and 3.

In order to evaluate the performance of Solution 2, we also perform simulations for the ARQ scheme with infinite number of feedback bits and the FEC scheme.

For each vehicle speed, the simulation results of the ARQ scheme with infinite number of feedback bits, the FEC scheme, and Solution 2 with the number of feedback bits=1, 2, and 3 are plotted in one figure in terms of PNSR vs bpp. This adds up to 15 curves in one figure for the three SNR values. To make the results easier to see and analyze, we also separately plot the curves for each SNR value.

\subsubsection{Simulation Results of Solution 2 at Vehicle Speed $=6 \mathrm{~km} / \mathrm{h}$}

The simulation results are plotted in Figure 4-1 to Figure 4-4. From these figures we can see that:

1. The performance of Solution 2 is always better than the performance of the FEC scheme.

2. The performance of Solution 2 gets closer and closer to the performance of the ARQ scheme with infinite number of feedback bits as the number of feedback bits increases.

3. At higher SNR values, Solution 2 can closely achieve the performance of the ARQ scheme with infinite number of feedback bits by a smaller number of feedback bits. In fact at $\mathrm{SNR}=14 \mathrm{~dB}$, using two feedback bits is enough to 
achieve a performance very close to that of infinite number of feedback bits.

At $20 \mathrm{~dB}$, only one feedback bit suffices for close to infinite feedback performance. This is because at higher SNR values, the performance of channel codes is much better.

4. The performances of ARQ scheme with infinite number of feedback bits at different SNRs are very close. As the number of feedback bits increases, the performance of Solution 2 becomes more robust with respect to the changes in the channel SNR. This is such that the performance of the ARQ scheme with infinite number of feedback bits is almost independent of SNR in the SNR range of interest and greatly dependent on the total bit budget. Note that this result may not hold for all rate-compatible channel codes and SNRs.

5. For a given bpp, the performance gap between the ARQ scheme with infinite number of feedback bits and the FEC scheme decreases as the SNR increases. As we know, the FEC scheme will stop receiving the bitstream when a decoding failure happens. At lower channel SNRs, the decoding failure happens earlier and therefore the performance of reconstruction image is worse. On the other hand, the performances of ARQ scheme with infinite number of feedback bits at different SNRs are almost the same.

6. For a given SNR, the performance gap between the ARQ scheme with infinite number of feedback bits and the FEC scheme increases as the total bit budget increases. 


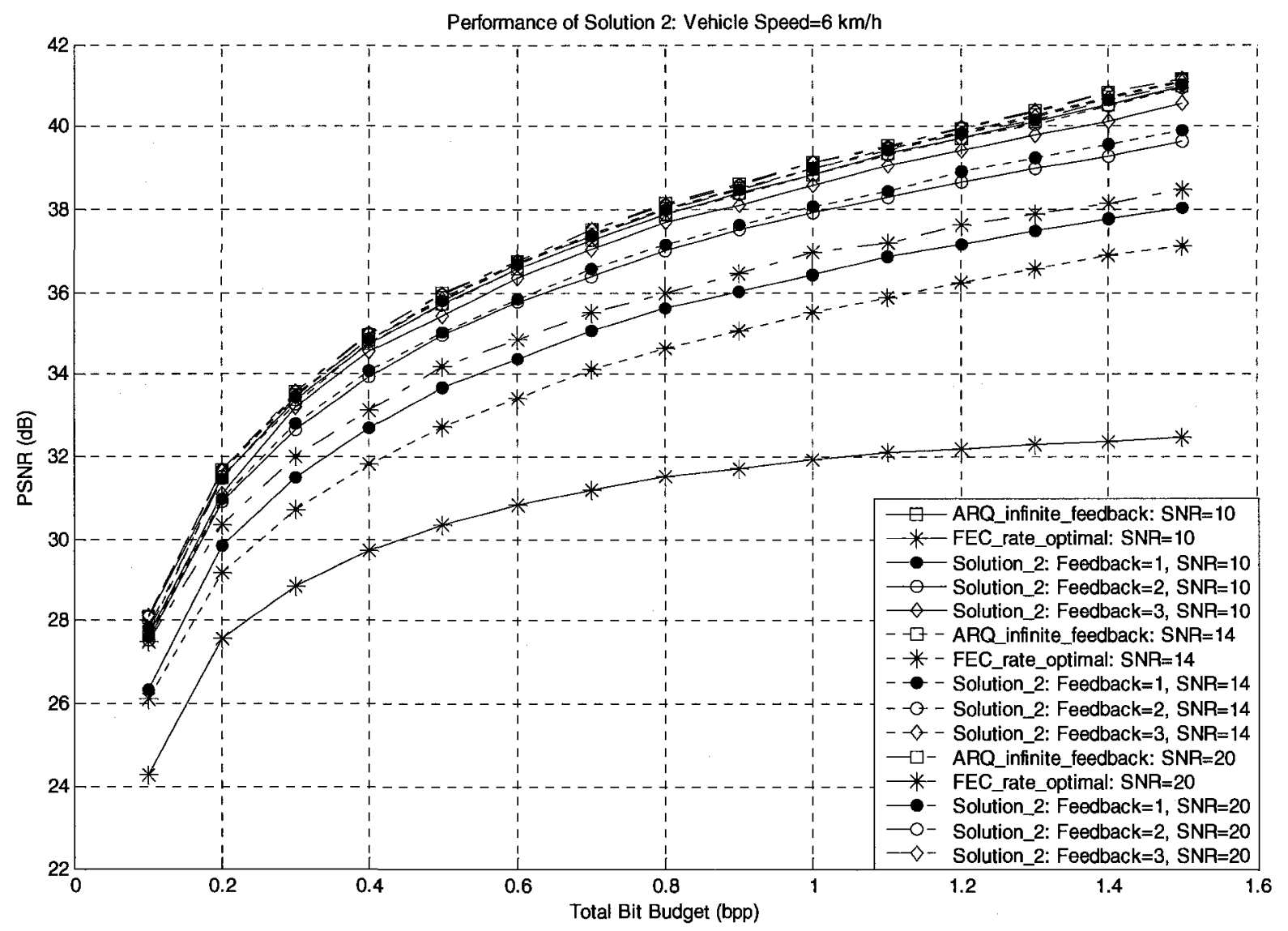

Figure 4-1 Performance of Solution 2: $V=6 \mathrm{~km} / \mathrm{h}, \mathrm{SNR}=10,14$, and $20 \mathrm{~dB}$ 


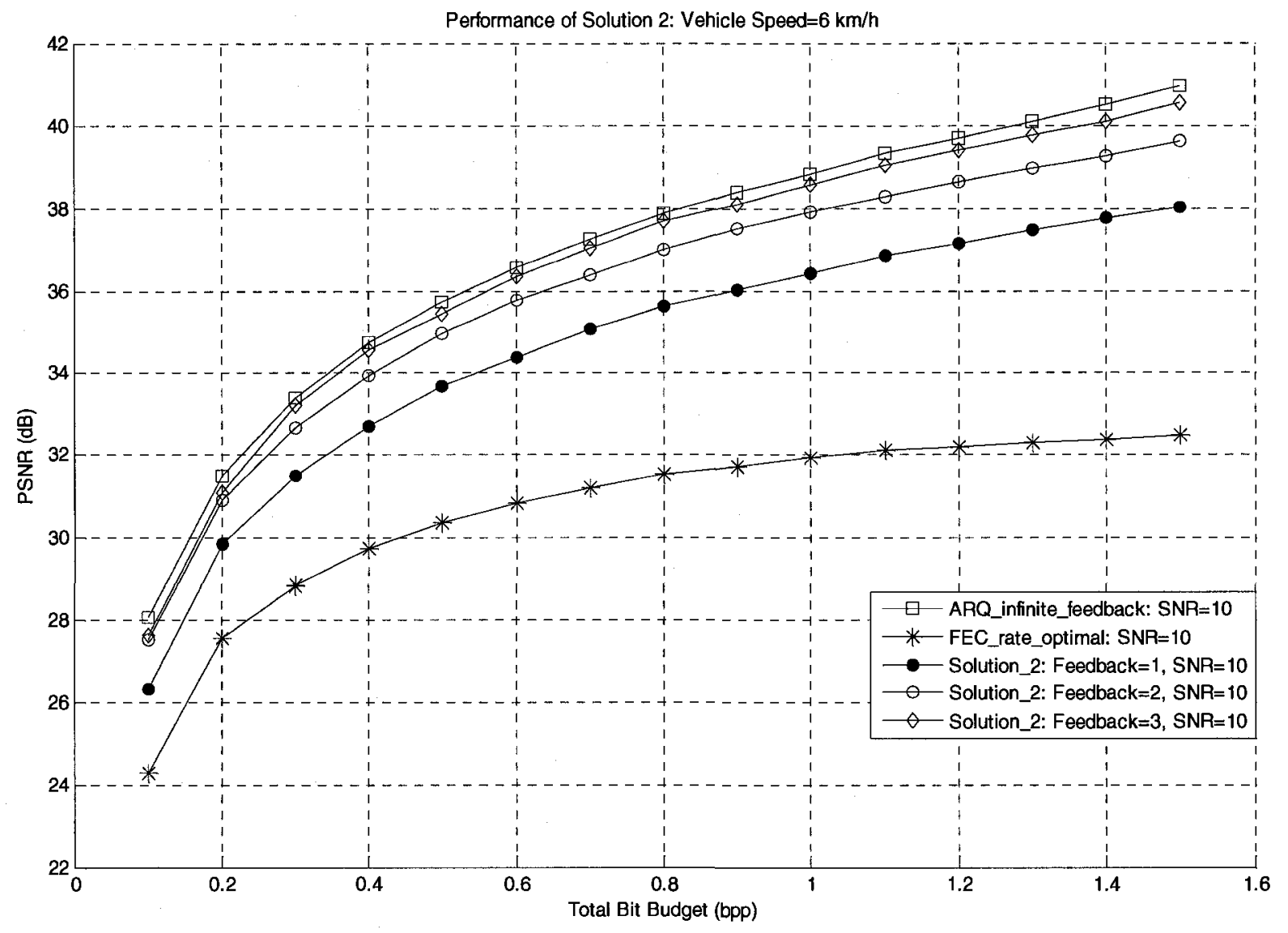

Figure 4-2 Performance of Solution 2: V=6 km/h, SNR=10 dB 


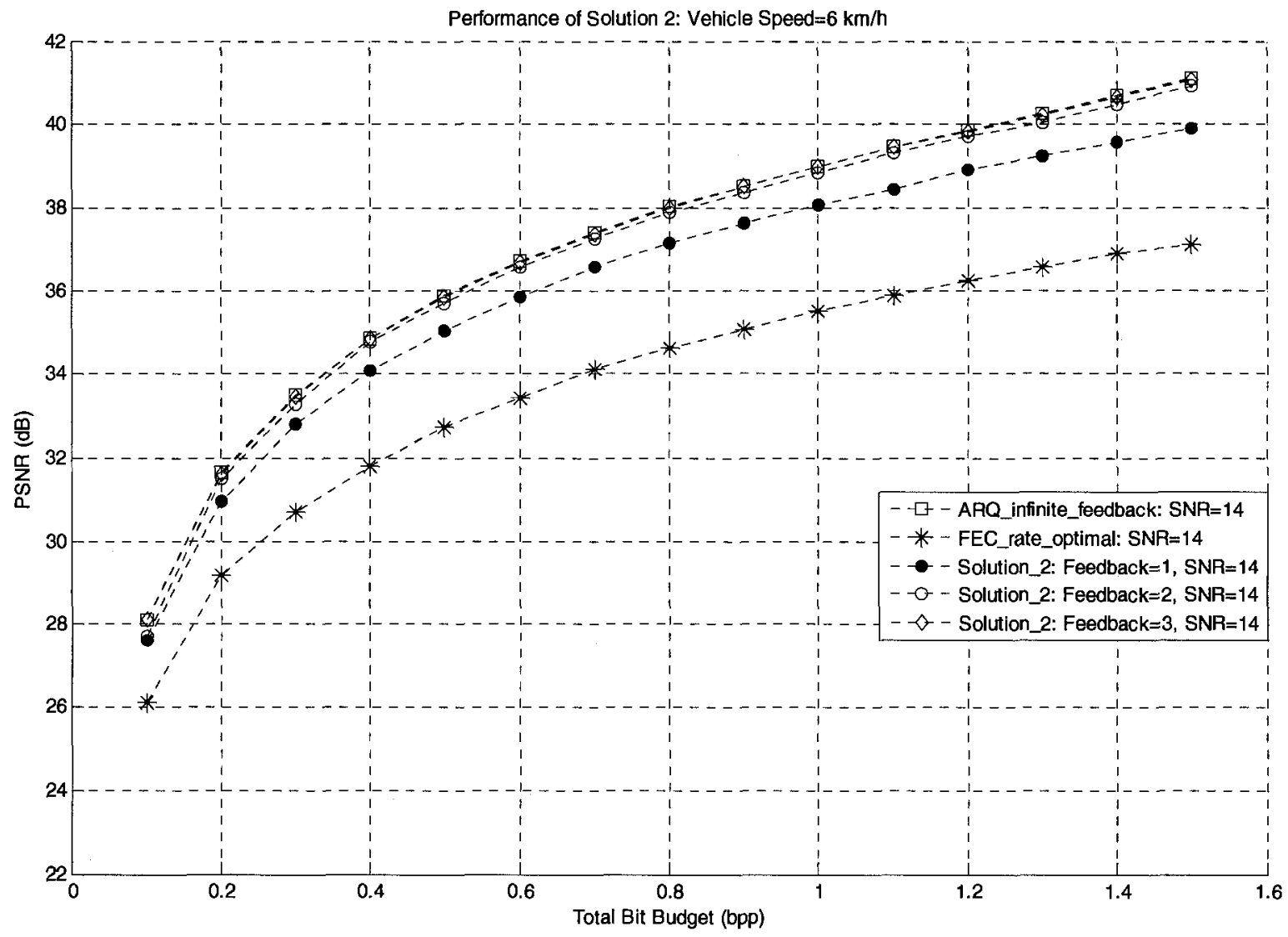

Figure 4-3 Performance of Solution 2: V=6 km/h, SNR=14 dB 


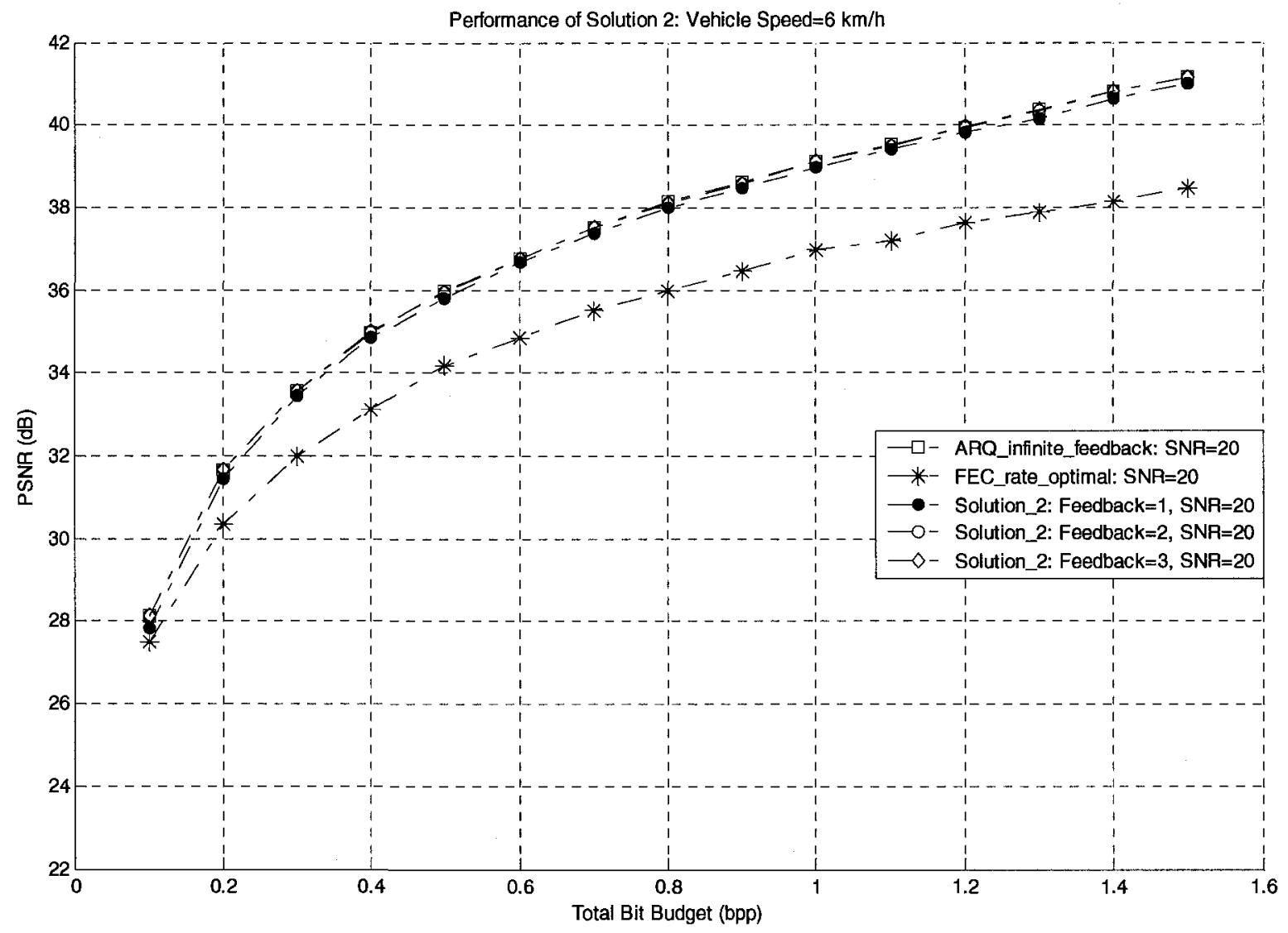

Figure 4-4 Performance of Solution 2: V=6 km/h, SNR=20 dB 


\subsubsection{Simulation Results of Solution 2 at Vehicle Speed=50 km/h}

The simulation results are plotted in Figure 4-5 to Figure 4-8. From the figures, we can draw the same conclusions as those of $V=6 \mathrm{~km} / \mathrm{h}$. As the speed increases the PSNR values at the same SNR and for the same total bit budget improve. This is due to the improvement in the error correcting performance of the channel codes. Also, for the same reason, by increasing the speed, the gap between the performance of the ARQ scheme with infinite number of feedback bits and the performance of the FEC scheme becomes smaller. Similarly the performance gap between infinite and finite feedback results decreases by increasing the speed. 


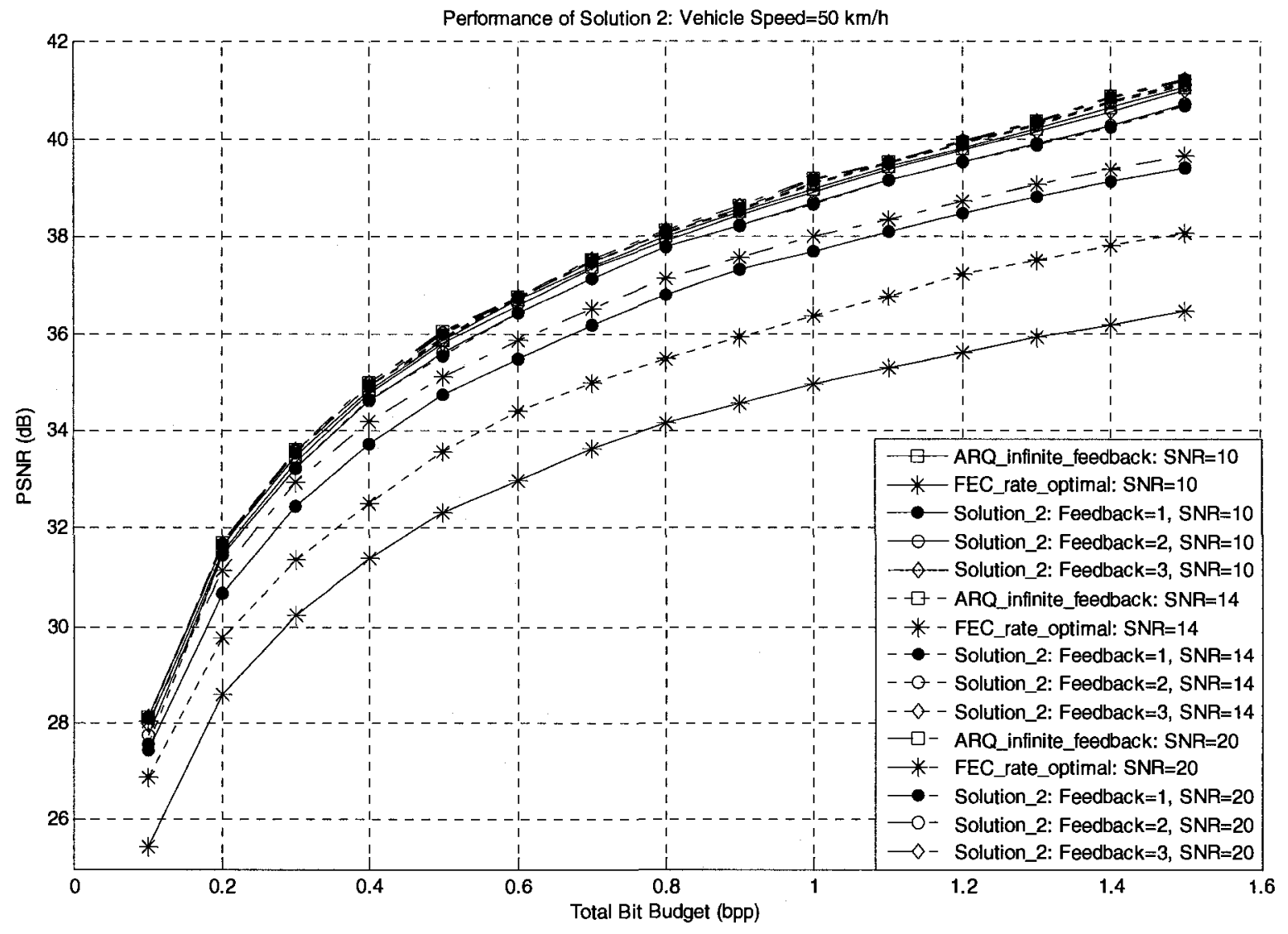

Figure 4-5 Performance of Solution 2: V=50 km/h, SNR=10, 14, and $20 \mathrm{~dB}$ 


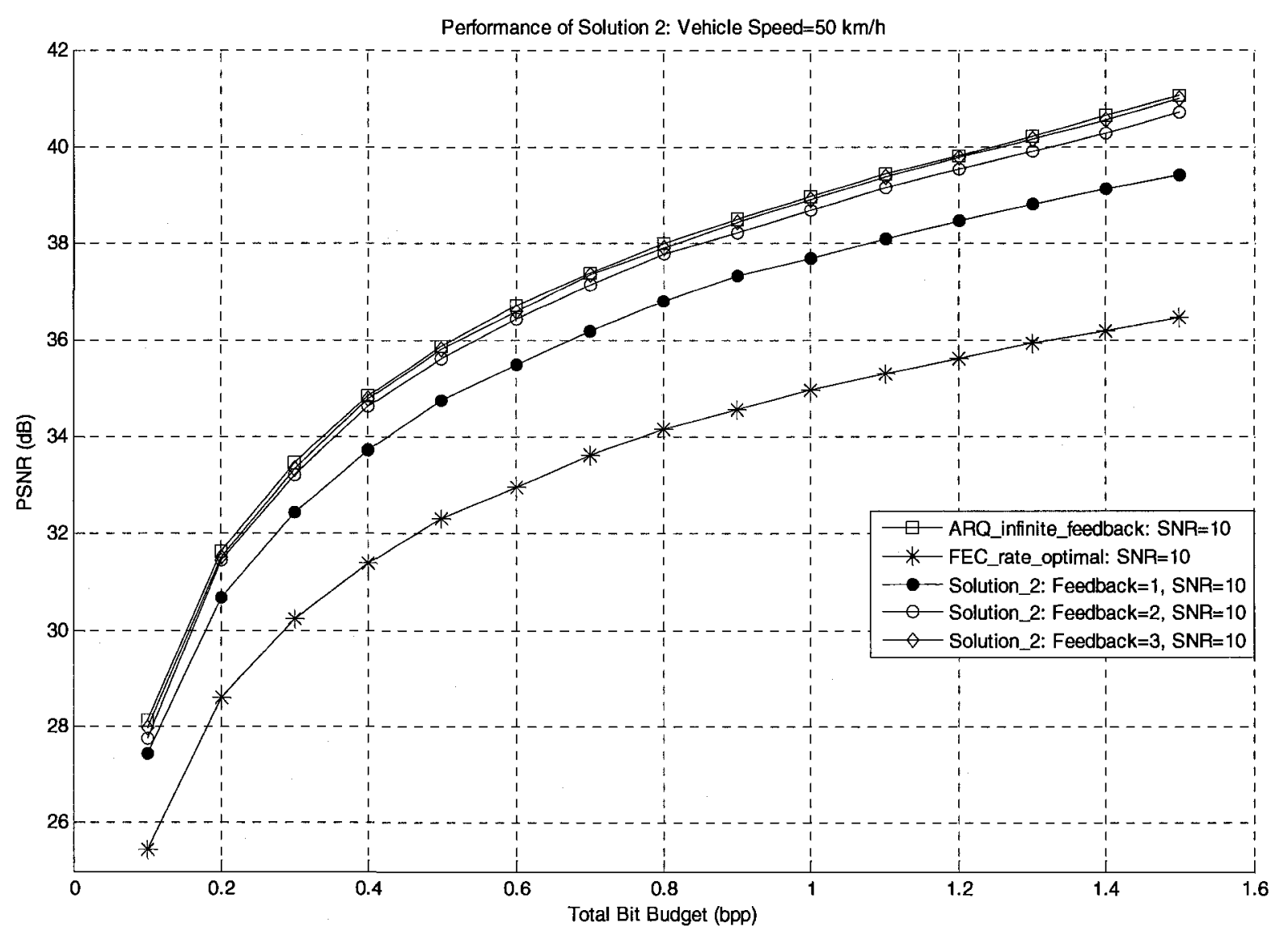

Figure 4-6 Performance of Solution 2: V=50 km/h, SNR=10 dB 


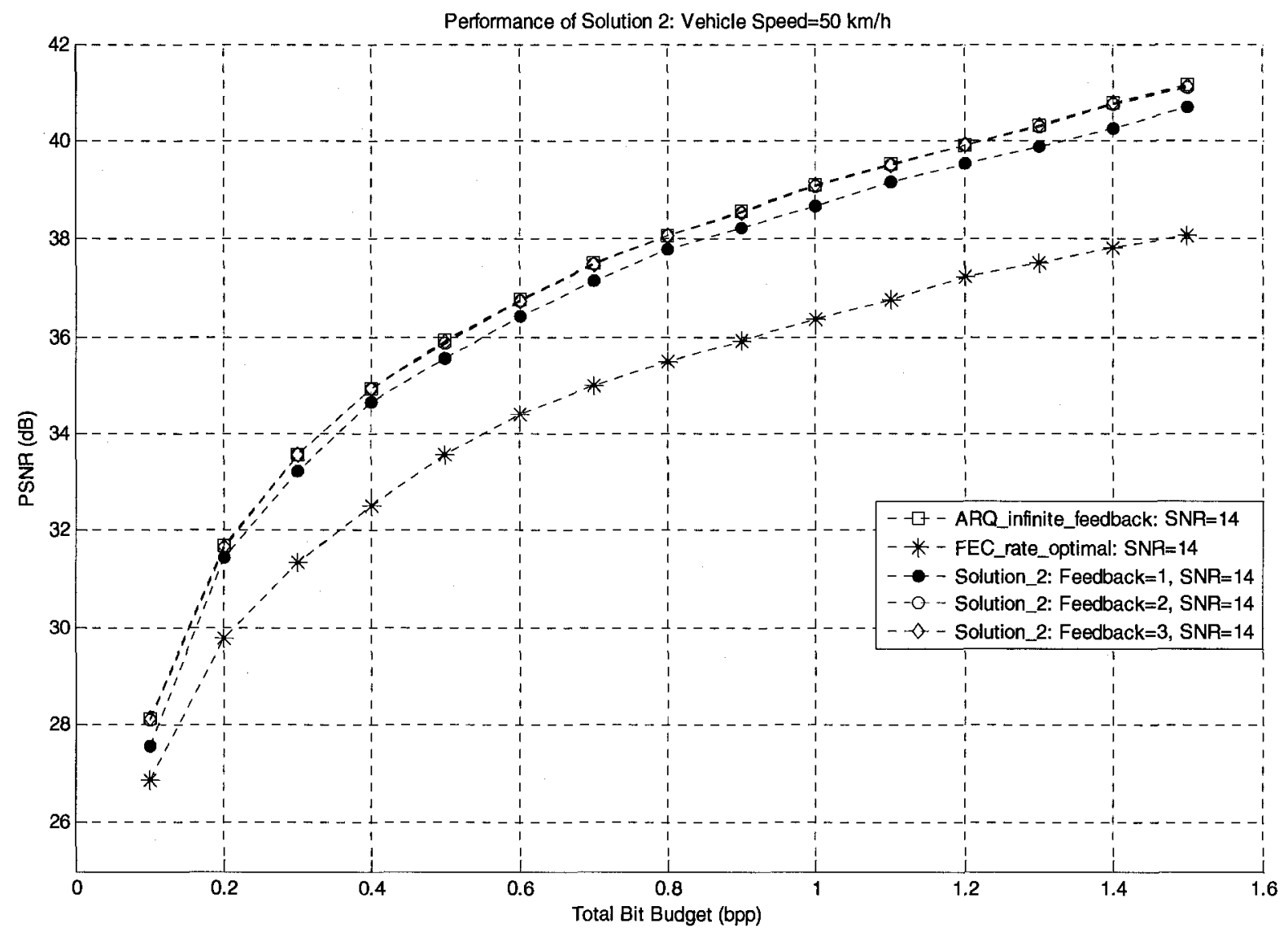

Figure 4-7 Performance of Solution 2: V=50 km/h, SNR=14 dB 


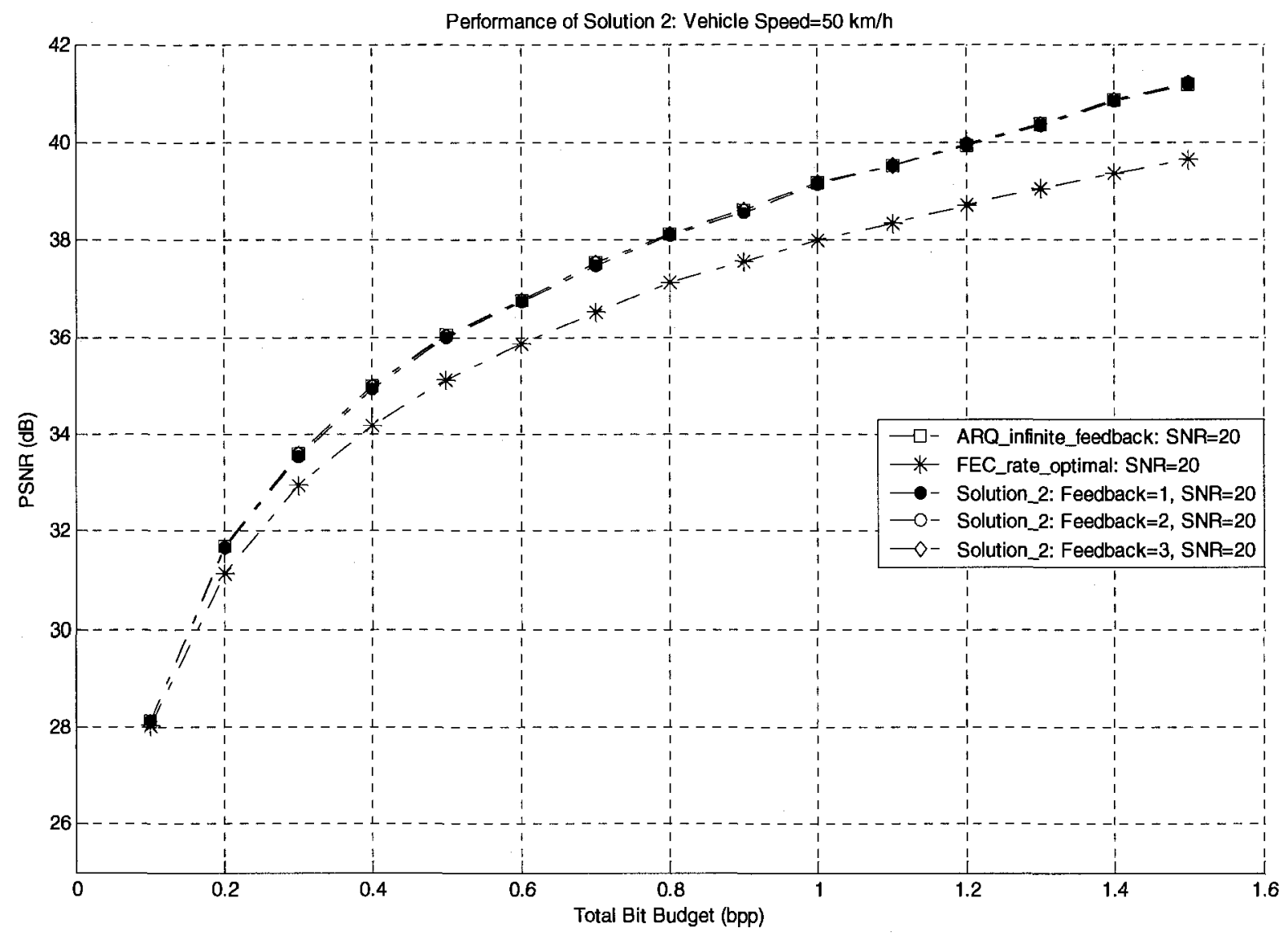

Figure 4-8 Performance of Solution $2: \mathrm{V}=50 \mathrm{~km} / \mathrm{h}, \mathrm{SNR}=20 \mathrm{~dB}$ 


\subsubsection{Simulation Results of Solution 2 at Vehicle Speed $=120 \mathrm{~km} / \mathrm{h}$}

The simulation results are plotted in Figure 4-9 to Figure 4-12. From the figures, we can draw the same conclusions as those discussed for $V=6 \mathrm{~km} / \mathrm{h}$ and $\mathrm{V}=50 \mathrm{~km} / \mathrm{h}$.

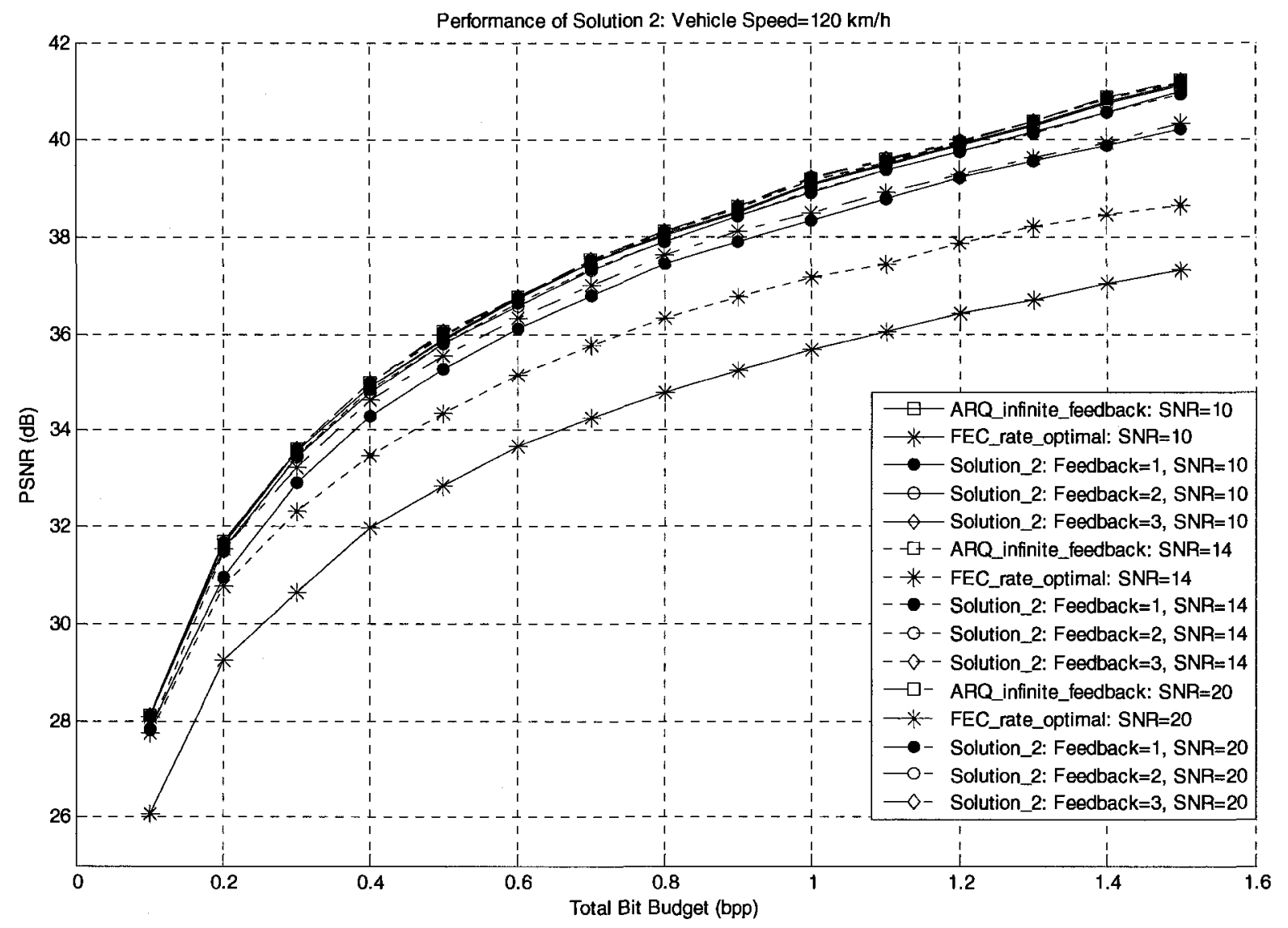

Figure 4-9 Performance of Solution 2: $\mathrm{V}=120 \mathrm{~km} / \mathrm{h}, \mathrm{SNR}=10,14$, and $20 \mathrm{~dB}$ 


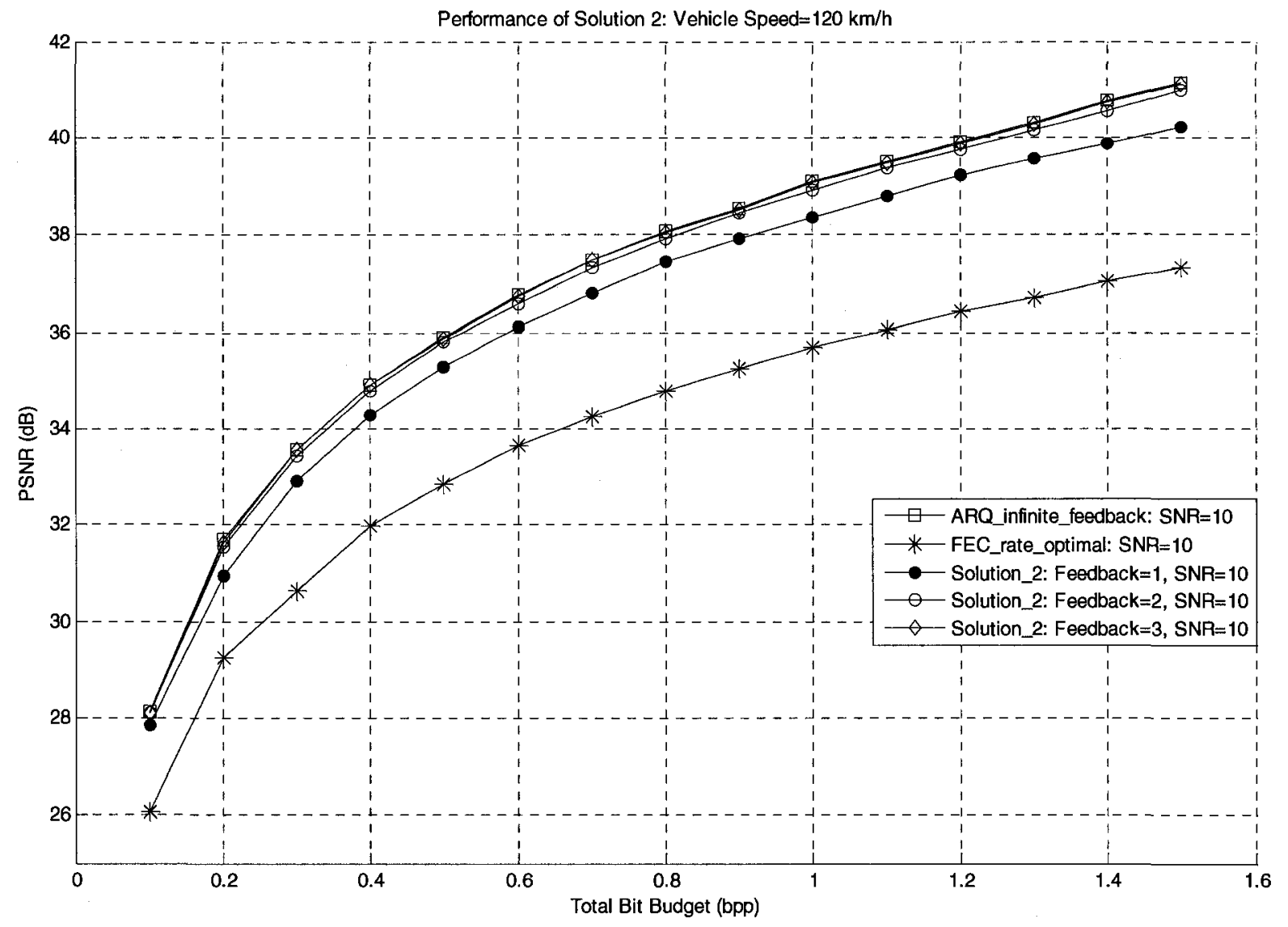

Figure 4-10 Performance of Solution 2: V=120 km/h, SNR=10 dB 


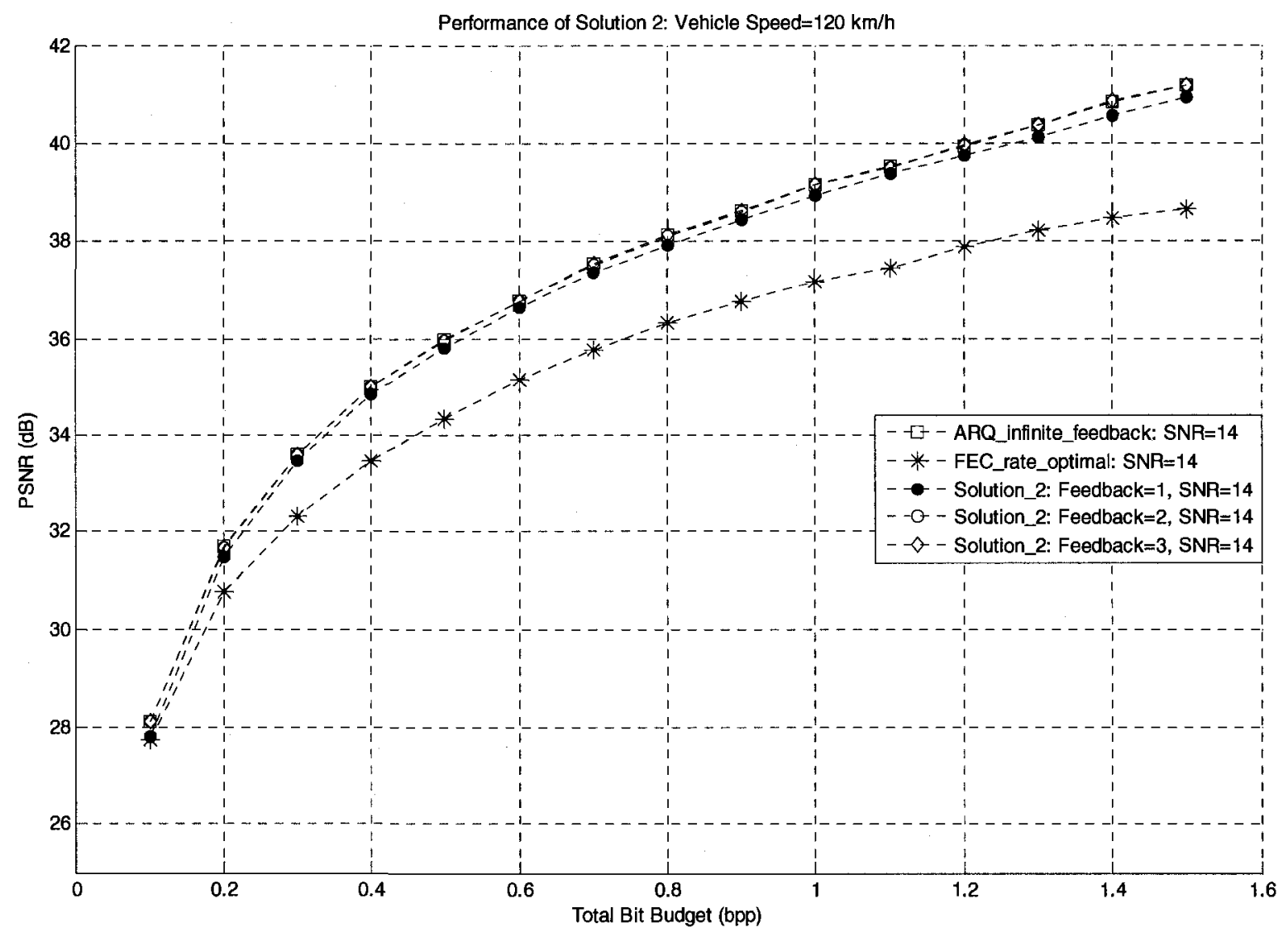

Figure 4-11 Performance of Solution 2: V=120 km/h, SNR=14 dB 


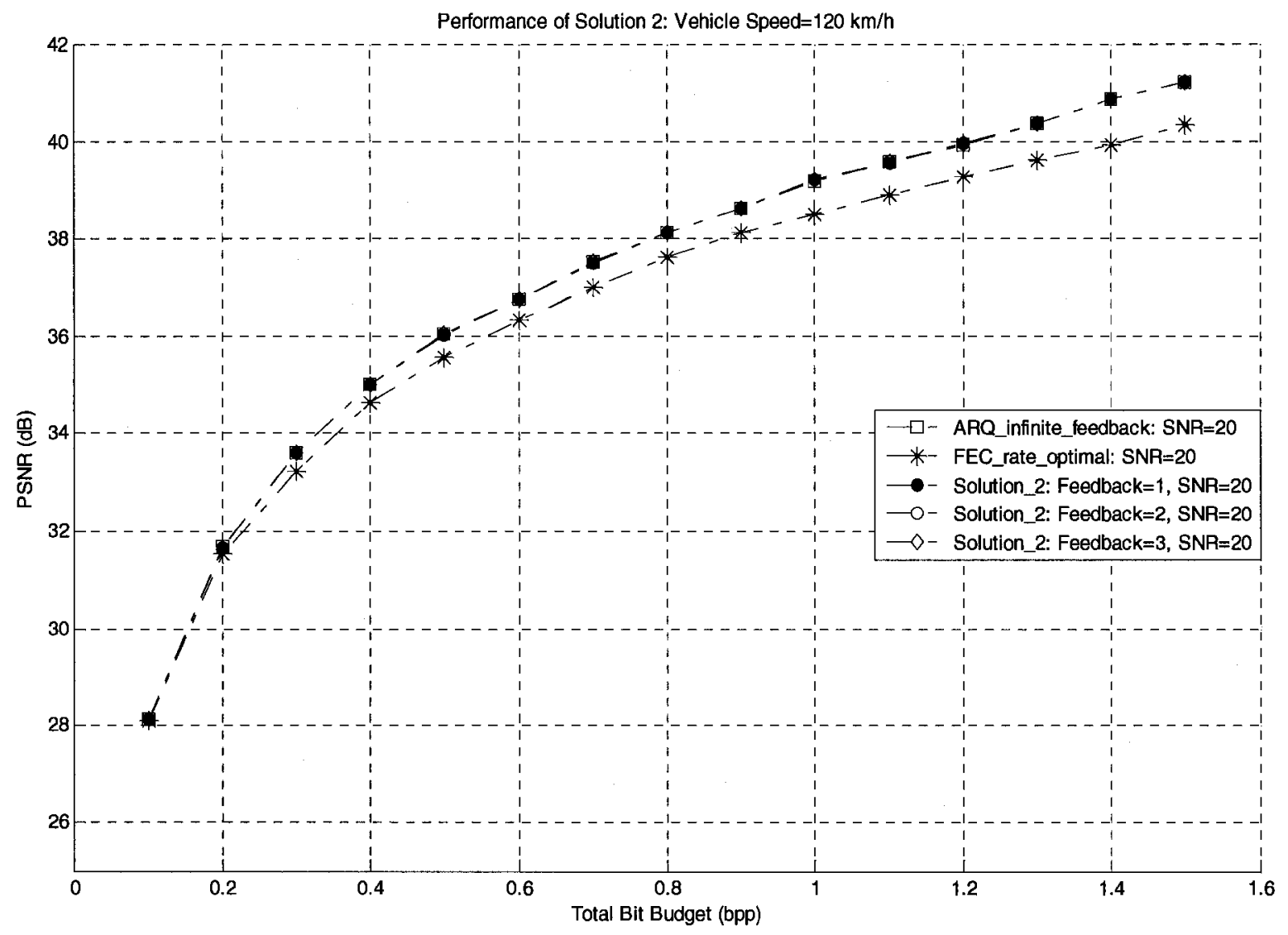

Figure 4-12 Performance of Solution 2: V=120 km/h, SNR=20 dB 


\subsection{Performance Comparison of Solution 2 and Solution 1}

In this section, we compare the performance of Solution 2 and Solution 1 in the correlated Rayleigh fading channels with SI at different vehicle speeds. For each vehicle speed, we obtain simulation results for $\mathrm{SNR}=10,14$, and $20 \mathrm{~dB}$ and total bit budget $=0.3$, $0.6,0.9,1.2$ and $1.5 \mathrm{bpp}$. In the simulations of Solution 1 , the threshold value $p_{e}$ is set to be 0.01 , as in [21]. But there is an exception, where $p_{e}=0.03$.

\subsubsection{Performance Comparison at Vehicle Speed $=6 \mathrm{~km} / \mathrm{h}$}

For $\mathrm{SNR}=10 \mathrm{~dB}$, we perform simulations with the number of feedback bits=1, 2 and 3 . The simulation results are shown in Table 4-5. From the table, we can find that Solution 2 achieves better performance than Solution 1 for all cases. For the number of feedback bits=1, the improvement of Solution 2 over Solution 1 is up to $1.24 \mathrm{~dB}$, and increases as the total bit budget becomes larger. For the number of feedback bits=2, the improvement of Solution 2 over Solution 1 is up to $\mathbf{0 . 3 4} \mathrm{dB}$, and increases as the total bit budget increases. For the number of feedback bits=3, the improvement of Solution 2 over Solution 1 is up to $\mathbf{0 . 1 5} \mathrm{dB}$. The simulation results are also plotted in Figure 4-13. From the figure, we can easily observe that the performance of Solution 2 is always better than the performance of Solution 1 for different number of feedback bits. As the number of feedback bits increases, the performance difference between Solution 2 and Solution 1 decreases, and the performances of both solutions get closer and closer to the performance of the ARQ scheme with infinite number of feedback bits. 
In Section 3.4, we mentioned that selecting an appropriate threshold value $p_{e}$ for the packet error rate is an important issue for Solution 1. An example of this can be seen in our simulations for $\mathrm{SNR}=10 \mathrm{~dB}$. The value of $p_{e}$ is set at 0.01 in [21]. This value however cannot be achieved for our system at $\mathrm{SNR}=10 \mathrm{~dB}$ since even the lowest rate code has a packet error probability larger than 0.01 . In such case, what should we do with Solution 1 ? Obviously, Solution 1 is a very coarse solution, far from the optimal solution. For the cases in which the lowest error probabilities that the channel codes can achieve is bigger than 0.01 (e.g. SNR=10), we try to use different $p_{e}$ values for Solution 1 and choose the largest PSNR value that the simulations can achieve as the final simulation result of Solution 1. To resolve this issue, we perform a search among all the achievable thresholds and find the one that results in the best end-to-end PSNR. At the SNR=10 dB, the value of $p_{e}$ is set at 0.03 .

For SNR=14 and $20 \mathrm{~dB}$, we perform simulations with the number of feedback bits=1. The simulation results are illustrated in Figure 4-14. From the figure, we can observe that the performance of Solution 2 is always better that the performance of Solution 1 although the advantage is not significant. The performances of two solutions are very close, especially at $\mathrm{SNR}=20 \mathrm{~dB}$. 


\begin{tabular}{|c|c|c|c|c|c|c|}
\hline \multirow{2}{*}{$\begin{array}{l}\text { Number of } \\
\text { Feedback } \\
\text { Bits }\end{array}$} & \multirow{2}{*}{ Solutions } & \multicolumn{5}{|c|}{ Total Bit Budget (bpp) } \\
\hline & & 0.3 & 0.6 & 0.9 & 1.2 & 1.5 \\
\hline$\infty$ & $\begin{array}{l}\text { Type II Hybrid ARQ } \\
\text { scheme with infinite } \\
\text { feedback bits }\end{array}$ & 33.39 & 36.58 & 38.41 & 39.73 & 40.97 \\
\hline 0 & FEC & 28.84 & 30.81 & 31.71 & 32.20 & 32.48 \\
\hline \multirow{3}{*}{1} & Solution 1 & 31.28 & 34.05 & 35.38 & 36.22 & 36.79 \\
\hline & Solution 2 & 31.48 & 34.36 & 36.04 & 37.15 & 38.03 \\
\hline & $\begin{array}{c}\text { Improvement } \\
\text { (Solution 2-Solution 1) }\end{array}$ & 0.20 & 0.31 & 0.66 & 0.93 & 1.24 \\
\hline \multirow{3}{*}{ 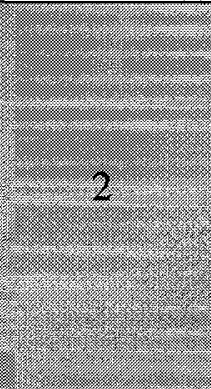 } & Solution 1 & 32.56 & 35.64 & 37.33 & 38.45 & 39.31 \\
\hline & Solution 2 & 32.67 & 35.75 & 37.53 & & \\
\hline & $\begin{array}{l}\text { Improvement } \\
\text { (Solution 2-Solution } 1 \text { ) }\end{array}$ & 0.11 & 0.11 & 0.20 & (2) & 0.34 \\
\hline \multirow{3}{*}{3} & Solution 1 & 33.05 & 36.24 & 38.04 & 39.33 & 40.41 \\
\hline & Solution 2 & 33.20 & 36.34 & 38.11 & 39.41 & 40.54 \\
\hline & $\begin{array}{c}\text { Improvement } \\
\text { (Solution 2-Solution 1) }\end{array}$ & 0.15 & 0.10 & 0.07 & 0.08 & 0.13 \\
\hline
\end{tabular}

Table 4-5 Performance comparison of Solution 2 and Solution 1 in $\mathrm{dB}: \mathrm{V}=6 \mathrm{~km} / \mathrm{h}$, $S N R=10 \mathrm{~dB}$ 


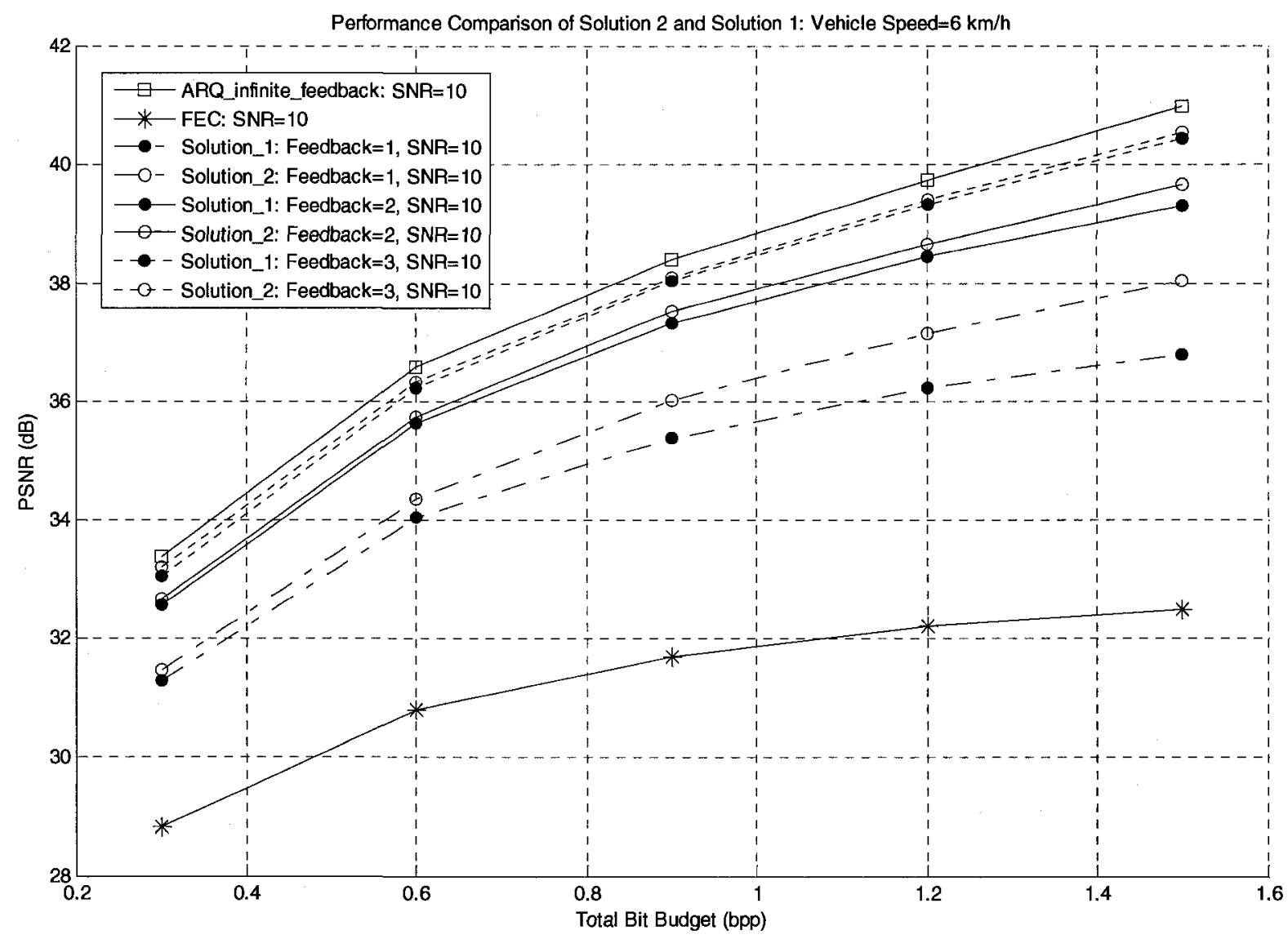

Figure 4-13 Performance comparison of Solution 2 and Solution 1: V=6 km/h, SNR=10 $\mathrm{dB}$ 


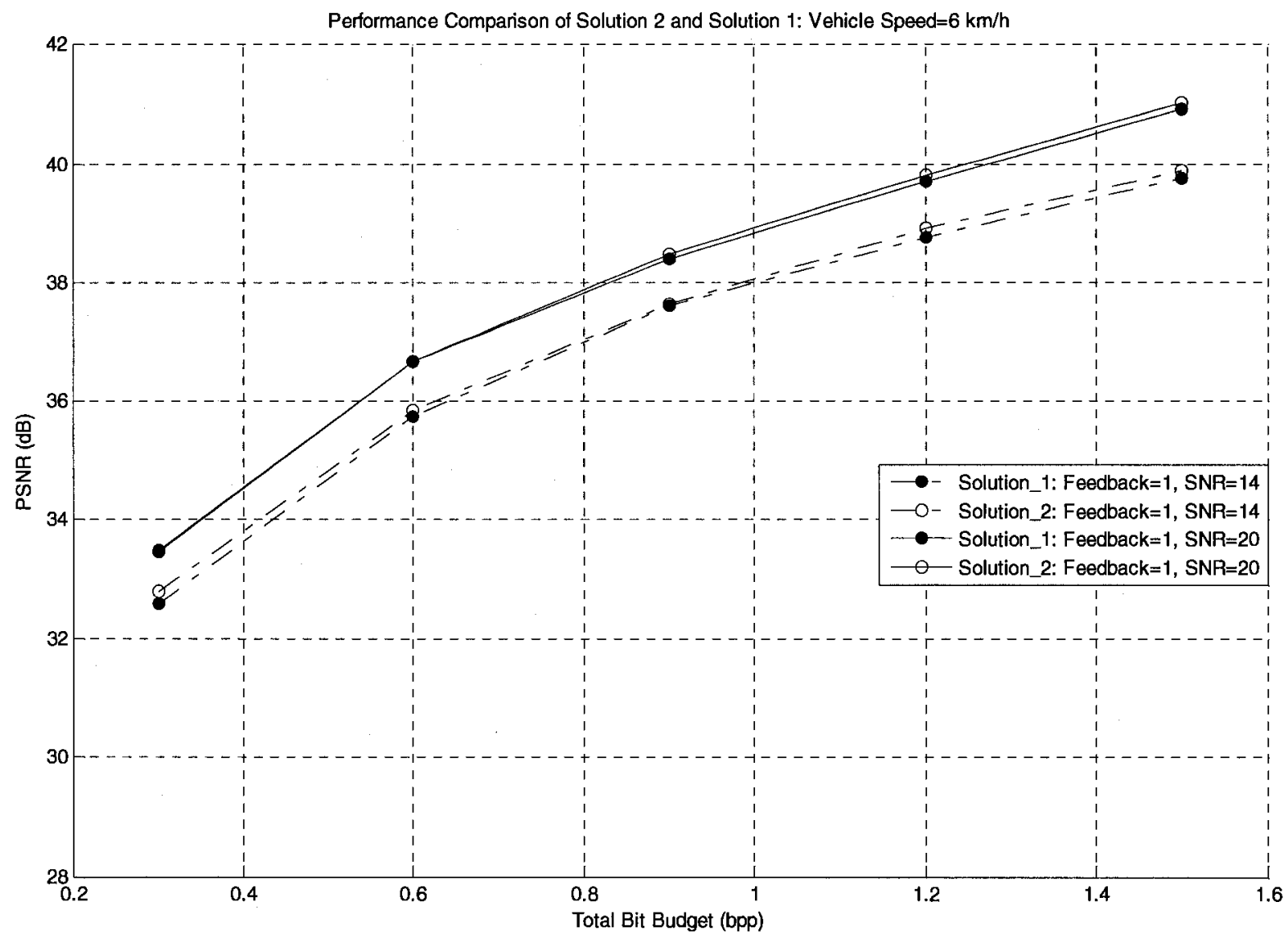

Figure 4-14 Performance comparison of Solution 2 and Solution $1: \mathrm{V}=6 \mathrm{~km} / \mathrm{h}, \mathrm{SNR}=14$ and $20 \mathrm{~dB}$ 


\subsubsection{Performance Comparison at Vehicle Speed $=50 \mathrm{~km} / \mathrm{h}$}

For vehicle speed $=50 \mathrm{~km} / \mathrm{h}$, we perform simulations with the number of feedback bits $=1$. The simulation results are shown in Table 4-6 and plotted in Figure 4-15. From Table 4-6, we can see that the performance of Solution 2 is better than the performance of Solution 1 for all cases except one. The performance improvement of Solution 2 over Solution 1 is up to $\mathbf{0 . 2 2} \mathrm{dB}$ at $\mathrm{SNR}=10$, and up to $\mathbf{0 . 3 0} \mathrm{dB}$ at $\mathrm{SNR}=14 \mathrm{~dB}$. At $\mathrm{SNR}=20$ $\mathrm{dB}$, the performances of two solutions are very close. For the exceptional case in which the performance of Solution 2 is worse than the performance of Solution 1 , the performance difference is only $\mathbf{0 . 0 1} \mathrm{dB}$, which is negligible. This is probably caused by the random characteristic of simulations and the fact that a solution achieving more useful source bits can not guarantee to achieve a higher PSNR value. From Figure 4-15, we can also observe that the performances of two solutions are very close at $\mathrm{SNR}=20 \mathrm{~dB}$. 


\begin{tabular}{|c|c|c|c|c|c|c|c|}
\hline \multirow{2}{*}{ SNR } & \multirow{2}{*}{$\begin{array}{l}\text { Number of } \\
\text { Feedback } \\
\text { Bits }\end{array}$} & \multirow{2}{*}{ Solutions } & \multicolumn{5}{|c|}{ Total Bit Budget (bpp) } \\
\hline & & & 0.3 & 0.6 & 0.9 & 1.2 & 1.5 \\
\hline \multirow{3}{*}{10} & \multirow{3}{*}{1} & Solution 1 & 32.21 & 35.41 & 37.23 & 38.35 & 39.30 \\
\hline & & Solution 2 & 32.43 & 35.50 & 37.31 & 38.47 & 39.39 \\
\hline & & $\begin{array}{c}\text { Improvement } \\
\text { (Solution 2- } \\
\text { Solution 1) }\end{array}$ & 0.22 & 0.09 & 0.08 & 0.12 & 0.09 \\
\hline \multirow{4}{*}{14} & \multirow{4}{*}{ 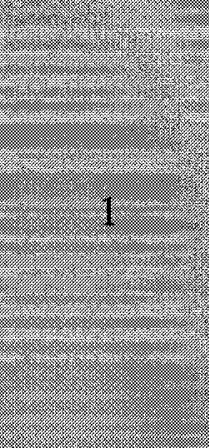 } & Solution 1 & 33.20 & 36.32 & 38.06 & 39.26 & 40.39 \\
\hline & & Solution 2 & 33.24 & 36.42 & 38.23 & 39.52 & 40,69 \\
\hline & & Improvement & & $5=$ & & $x^{2}=0$ & \\
\hline & & $\begin{array}{l}\text { (Solution 2- } \\
\text { Solution 1) }\end{array}$ & 0.01 & 0.10 & 0.17 & 0.26 & 0.30 \\
\hline \multirow{3}{*}{20} & \multirow{3}{*}{1} & Solution 1 & 33.57 & 36.74 & 38.61 & 39.89 & 41.15 \\
\hline & & Solution 2 & 33.58 & 36.73 & 38.62 & 39.92 & 41.17 \\
\hline & & $\begin{array}{c}\text { Improvement } \\
\text { (Solution 2- } \\
\text { Solution 1) }\end{array}$ & 0.01 & -0.01 & 0.01 & 0.03 & 0.02 \\
\hline
\end{tabular}

Table 4-6 Performance comparison of Solution 2 and Solution 1: V=50 km/h, SNR=10, 14 and $20 \mathrm{~dB}$ 


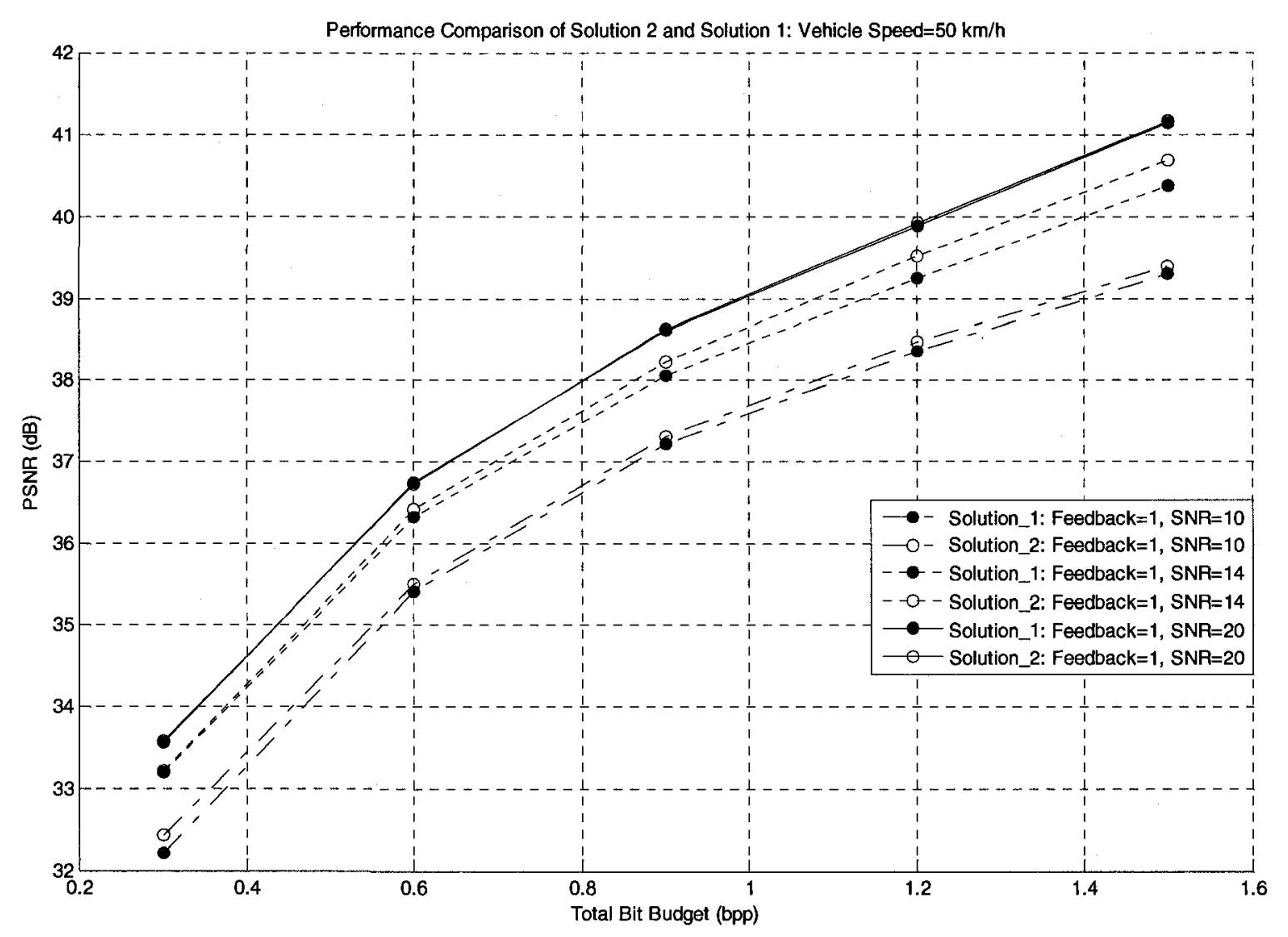

Figure 4-15 Performance comparison of Solution 2 and Solution 1: V=50 km/h, $\mathrm{SNR}=10,14$ and $20 \mathrm{~dB}$ 


\subsubsection{Performance Comparison at Vehicle Speed $=120 \mathrm{~km} / \mathrm{h}$}

For vehicle speed $=120 \mathrm{~km} / \mathrm{h}$, we perform simulations with the number of feedback bits $=1$. The simulation results are shown in Table 4-7 and plotted in Figure 4-16. From Table 4-7, we can see that the performance of Solution 2 is better than the performance of Solution 1 for all cases except one. The performance improvement of Solution 2 over Solution 1 is up to $\mathbf{0 . 1 1} \mathrm{dB}$ at $\mathrm{SNR}=10 \mathrm{~dB}, \mathbf{0 . 1 2} \mathrm{dB}$ at $\mathrm{SNR}=14 \mathrm{~dB}$ and $0.09 \mathrm{~dB}$ at $\mathrm{SNR}=20 \mathrm{~dB}$. For the exceptional case in which the performance of Solution 2 is worse than the performance of Solution 1 , the performance difference is only $\mathbf{0 . 0 1} \mathrm{dB}$, which is negligible and probably caused by the random characteristic of simulations. From Figure 4-16, we can also observe that the performances of two solutions are very close at $\mathrm{SNR}=20 \mathrm{~dB}$. 
63

\begin{tabular}{|c|c|c|c|c|c|c|c|}
\hline \multirow{2}{*}{ SNR } & \multirow{2}{*}{$\begin{array}{c}\text { Number of } \\
\text { Feedback } \\
\text { Bits }\end{array}$} & \multirow{2}{*}{ Solutions } & \multicolumn{5}{|c|}{ Total Bit Budget (bpp) } \\
\hline & & & 0.3 & 0.6 & 0.9 & 1.2 & 1.5 \\
\hline \multirow{4}{*}{10} & 0 & FEC & 30.65 & 33.66 & 35.24 & 36.41 & 37.31 \\
\hline & \multirow{3}{*}{1} & Solution 1 & 32.84 & 36.06 & 37.84 & 39.10 & 40.11 \\
\hline & & Solution 2 & 32.91 & 36.10 & 37.92 & 39.20 & 40.22 \\
\hline & & $\begin{array}{c}\text { Improvement } \\
\text { (Solution 2- } \\
\text { Solution 1) }\end{array}$ & 0.07 & 0.04 & 0.08 & 0.10 & 0.11 \\
\hline \multirow{4}{*}{14} & 0 & FEC & 32.32 & 35.14 & 36.77 & 37.88 & 38.67 \\
\hline & \multirow{3}{*}{1} & Solution 1 & 33.48 & 36.62 & 38.37 & 39.64 & 40.82 \\
\hline & & Solution 2 & 33.47 & 36.65 & 38.44 & 39.76 & 40.94 \\
\hline & & $\begin{array}{c}\text { Improvement } \\
\text { (Solution 2- } \\
\text { Solution 1) }\end{array}$ & -0.01 & 0.03 & 0.07 & 0.12 & 0.12 \\
\hline \multirow{4}{*}{20} & 0 & FEC & 33.22 & 36.33 & 38.12 & 39.28 & 40.33 \\
\hline & \multirow{3}{*}{1} & Solution 1 & 33.55 & 36.72 & 38.58 & 39.88 & 41.13 \\
\hline & & Solution 2 & 33.59 & 36.75 & 38.62 & 39.97 & 41.22 \\
\hline & & $\begin{array}{c}\text { Improvement } \\
\text { (Solution 2- } \\
\text { Solution 1) }\end{array}$ & 0.04 & 0.03 & 0.04 & 0.09 & 0.09 \\
\hline
\end{tabular}

Table 4-7 Performance comparison of Solution 2 and Solution $1: \mathrm{V}=120 \mathrm{~km} / \mathrm{h}$, $\mathrm{SNR}=10,14$ and $20 \mathrm{~dB}$ 


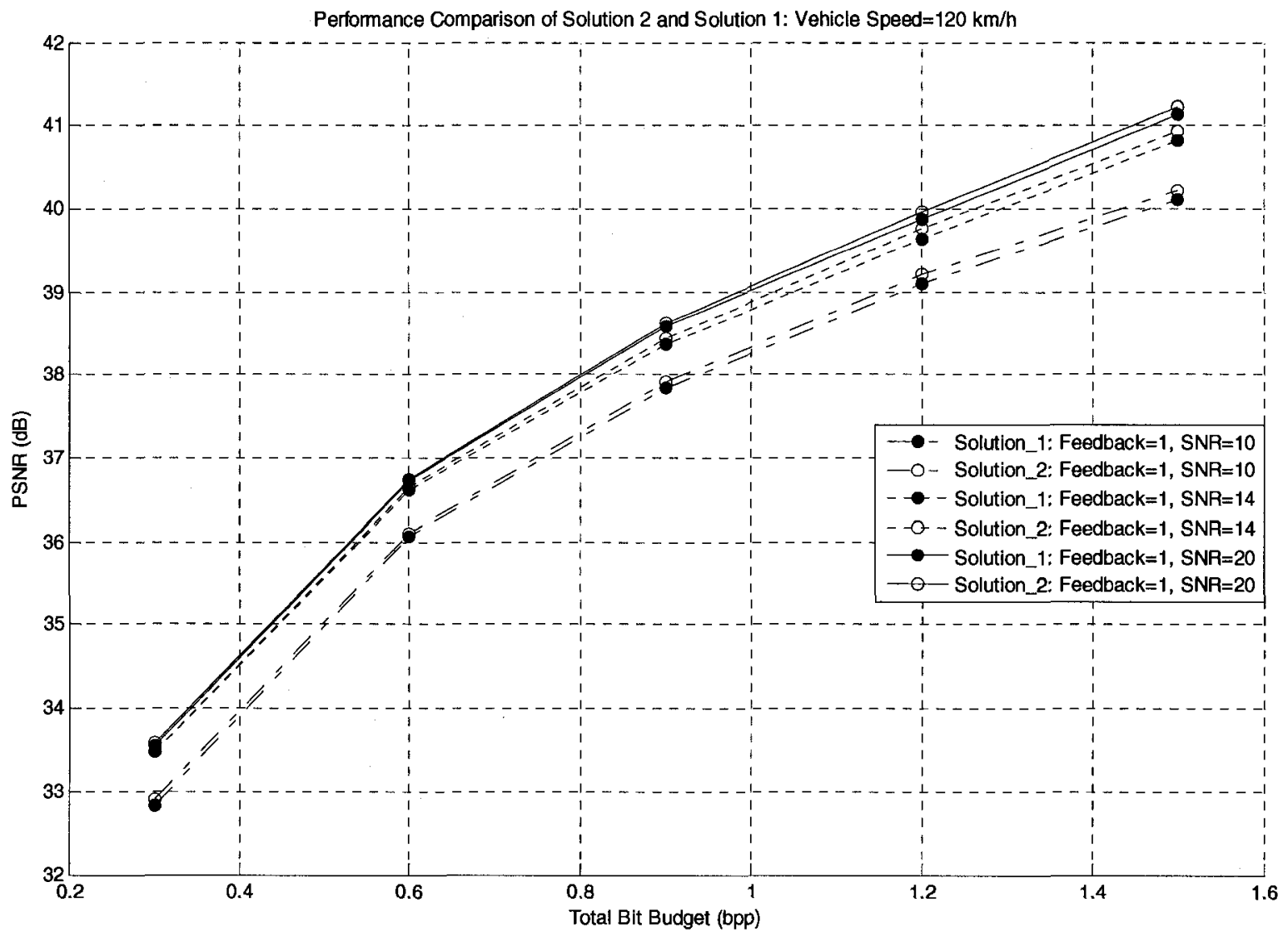

Figure 4-16 Performance comparison of Solution 2 and Solution 1: V=120 km/h, $\mathrm{SNR}=10,14$ and $20 \mathrm{~dB}$ 


\subsection{Performance Comparison of Solution 2 and Solution 3}

In this section, we compare the performance of Solution 2 and Solution 3 in the correlated Rayleigh fading channels with SI. For vehicle speed $=6 \mathrm{~km} / \mathrm{h}$, we obtain simulation results for $\mathrm{SNR}=10,14$, and $20 \mathrm{~dB}$ and total bit budget=0.2, 0.4, 0.6, 0.8 and $1.0 \mathrm{bpp}$. The number of feedback bits for each packet is considered to be 1 . As discussed in Chapter 3, Solution 2 is rate-optimal solution and Solution 3 is distortion-optimal solution. For Solution 3, both MSE and PSNR measurements are considered in simulations. So at each SNR value, 3 performances are simulated: the performance of Solution 2, the performance of Solution 3 with MSE measurement, and the performance of Solution 3 with PSNR measurement.

The simulation results are illustrated in Figure 4-17. In this figure, there are actually 9 curves (there are 3 different SNR values and each SNR value has 3 curves), but it seems there are only 3 curves. This is because for each SNR value, the performance of Solution 2, the performance of Solution 3 with MSE measurement and the performance of Solution 3 with PSNR measurement are very close, and therefore the 3 different curves look almost like one curve.

Although the performance of Solution 2, the performance of Solution 3 with MSE measurement and the performance of Solution 3 with PSNR measurement are very close, we still can tell the small difference between any two of them. In general, the performance of Solution 2 is better than the performance of Solution 3 with MSE measurement, but worse than the performance of Solution 3 with PSNR measurement. We can see this by observing Figure 4-18, which is the enlarged figure for $S N R=10 \mathrm{~dB}$. 
Since the performance of Solution 2 is very close to the performance of Solution 3, while the computational complexity of Solution 2 is much lower than that of Solution 3, we can simply use Solution 2 to get the optimal policies for progressive image transmission with feedback.

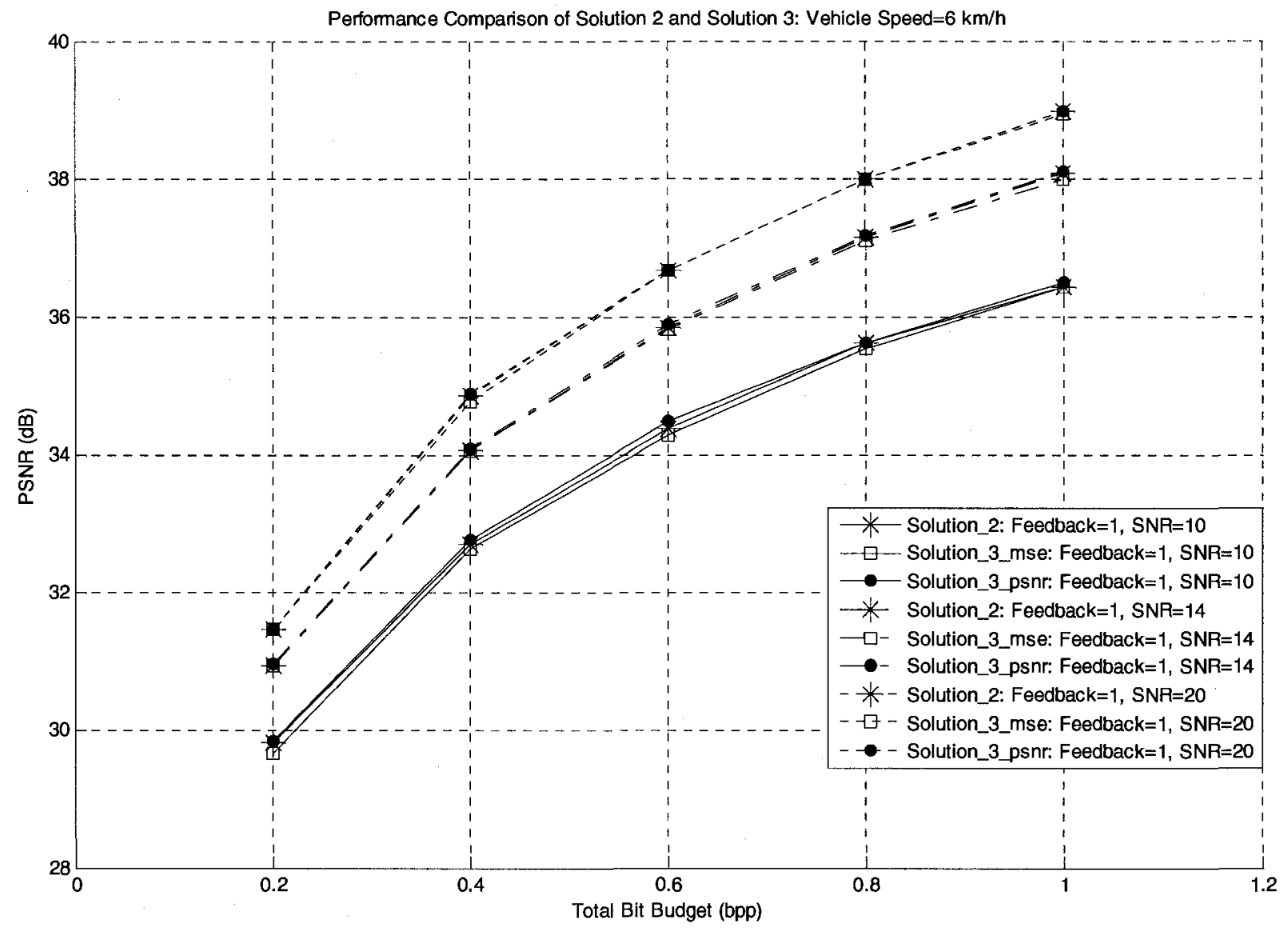

Figure 4-17 Performance comparison of Solution 2 and Solution 3: V=6 km/h, $\mathrm{SNR}=10,14$ and $20 \mathrm{~dB}$ 


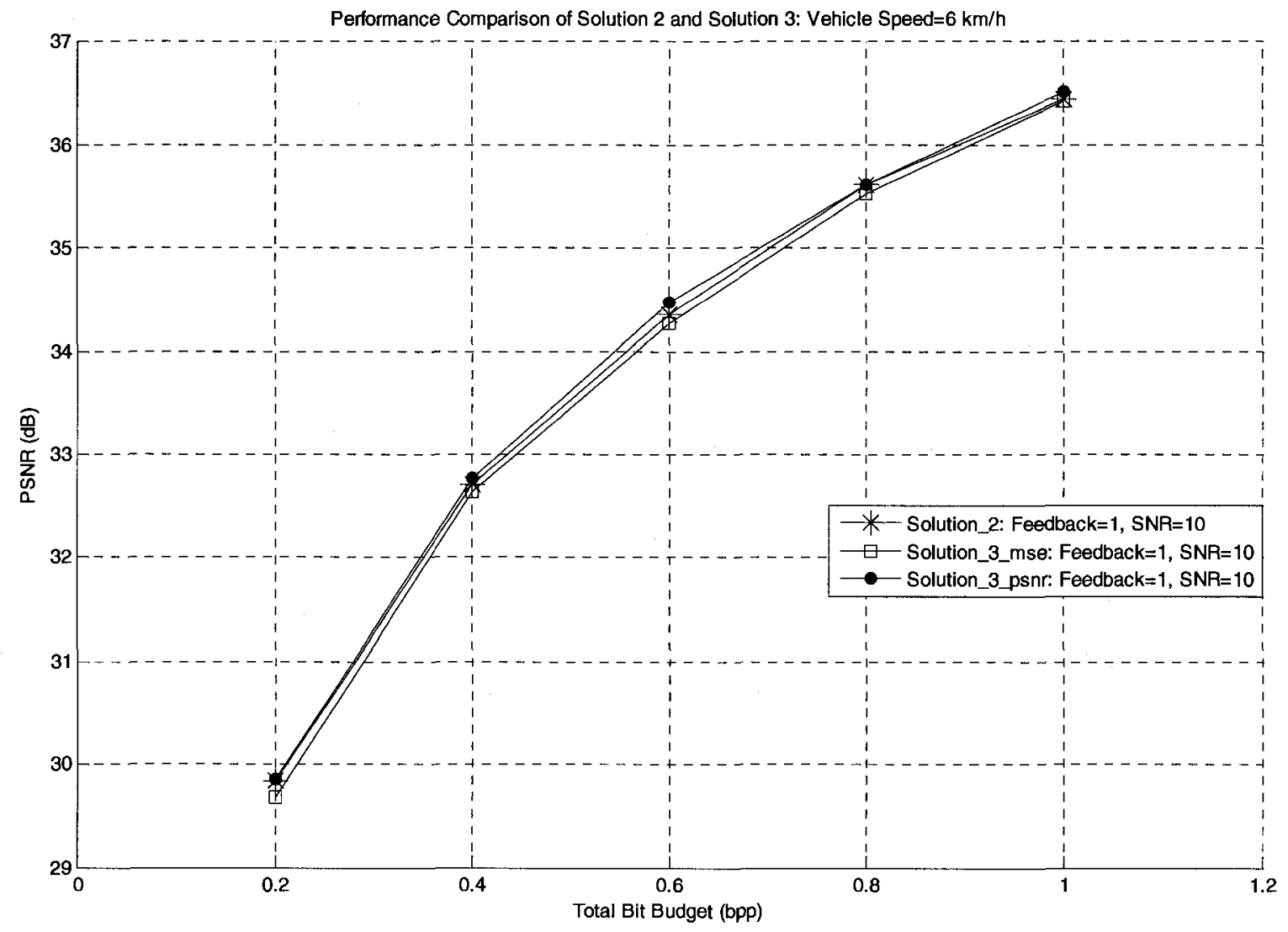

Figure 4-18 Performance comparison of Solution 2 and Solution 3: V=6 km/h, $\mathrm{SNR}=10 \mathrm{~dB}$ 


\subsection{Summary for the Simulation Results}

The performance comparison of Solution 2 and Solution 1 demonstrates that Solution 2 has better performance than Solution 1. In general, at low SNR or large total bit budget, the advantage of Solution 2 is more prominent. From the simulation results of Solution 1, we can see that Solution 1 is a coarse solution, far from optimal. Another disadvantage of Solution 1 is the difficulty in the appropriate selection of threshold value $p_{e}$.

On the contrary, Solution 2 is a solution, which is applicable to any situation and can achieve a better performance than Solution 1 .

In Section 4.5, the performance comparison of Solution 2 and Solution 3 demonstrates that the performance of Solution 2 and the performance of Solution 3 are very close.

We have also conducted the simulations using other standard test images such as Goldhill and Barbara (with gray scale, 8 bits per pixel, and dimensions $512 \times 512$ ) and get the similar results as using Lena. 


\section{Chapter 5 Conclusions and Future Work}

\subsection{Conclusions}

In this thesis, we investigate the rate allocation problem of progressive image transmission over noisy channels with feedback and propose some solutions. For the scenario of feedback constraint on each packet, both rate-optimal and distortion-optimal solutions are discussed. Solution 2 is based on rate-optimal and Solution 3 is based on distortion-optimal. In the proposed solutions, we apply the cost functions of FEC scheme to ARQ scheme, by introducing the concepts of average number of transmission bits and average error probability for a policy. In order to reduce the computational complexity of the solutions, three measures are used to reduce the number of candidate policies as well as the number of states at each level when we use either forward Viterbi Algorithm or backward dynamic programming approach to implement the solutions. These measures are: (1). For two possible policies $\pi_{1}$ and $\pi_{2}$, if the average number of transmission bits of $\pi_{2}$ is greater than that of $\pi_{1}$, but the average error probability of $\pi_{2}$ is not smaller than that of $\pi_{1}$, then $\pi_{2}$ will be eliminated from the set of candidate policies and $\pi_{1}$ will remain. If $\pi_{1}$ and $\pi_{2}$ have the same average error probabilities but different average number of transmission bits, then the policy with a greater average number of transmission bits will be eliminated and the other will remain. (2). When the average error probability of a policy is less than $1 \times 10^{-5}$, it will be rounded to 0 . (3). The average number of transmission bits is rounded to the nearest integer. We demonstrate that these 
simplification measures are effective and efficient in reducing the computational complexity of the proposed solutions. We also demonstrate that our proposed solutions achieve a better end-to-end performance than the existing solution in [21].

By comparing the performances of our proposed solutions, ARQ scheme with infinite number of feedback bits and FEC scheme, we find that: (1). The performance of our proposed solutions is always better than the performance of the FEC scheme. (2). The performance of our proposed solutions get closer and closer to the performance of ARQ scheme with infinite number of feedback bits as the number of feedback bits increases. (3). At higher SNR values, our solutions can closely achieve the performance of the ARQ scheme with infinite number of feedback bits by a smaller number of feedback bits. (4). The performance of ARQ scheme with infinite number of feedback bits at different SNR values are very close. (5). For a given total bit budget, the performance gap between the ARQ scheme with infinite number of feedback bits and the FEC scheme decreases as the SNR increases. (6). For a given SNR, the performance gap between the ARQ scheme with infinite number of feedback bits and the FEC scheme increases as the total bit budget increases.

Since our proposed solutions are based on the cost functions, they have a higher complexity than the existing solution in [21], in which an approximate approach is used to find a policy and apply this policy to all packets.

Although the simulations are performed with the JPEG2000 source code and RCLDPC channel codes, our proposed solutions are applicable to any source code, any ratecompatible channel codes and any channel conditions as long as the distortion-rate 
function of the source code, the total transmission bits and the error probabilities of the channel codes under the desirable channel conditions are all given.

\subsection{Future Work}

A few suggestions on future research are presented below.

1. The rate-allocation problem with a constraint on the total number of feedback bits may be interesting in some applications. Formulating and solving this problem would be of interest.

2. Investigation of rate allocation problem for MIMO-OFDM systems with feedback would be interesting.

3. Investigation of rate allocation problem for progressive video transmission over noisy channel with feedback would be interesting. Please refer to [9], [31].

4. Investigation of rate allocation problem for progressive image transmission over noisy channel with feedback by channel estimation would be also interesting. Please refer to [32]. 


\section{References}

[1] D. Taubman and M. Marcellin, JPEG2000: Image Compression Fundamentals, Standards, and Practice, Norwell, MA: Kluwer, 2002.

[2] JPEG2000 Standard [online]: http://www.jpeg.org/jpeg2000/index.html.

[3] A. Said and W. Pearlman, "A new, fast, and efficient image codec based on set partitioning in hierarchical trees," IEEE Transactions on Circuits and Systems for Video Technology, Vol. 6, No. 3, pp. 243-250, June 1996.

[4] R. Hamzaoui, V. Stankovic and Z. Xiong, "Optimized error protection of scalable image bit streams," IEEE Signal Processing Magazine, Vol. 22, No. 6, pp. 91-107, November 2005.

[5] S. Lin and D.J. Costello. Jr., Error Control Coding: Fundamentals and Applications. Englewood Cliffs, NJ: Prentice Hall, 1983.

[6] S. Lin, D.J. Costello Jr., and M.J. Miller, "Automatic repeat-request error control schemes," IEEE Communication Magazine, Vol. 12, pp. 5-17, December 1984.

[7] A. Nosratinia, J. Lu, and B. Aazhang, "Source-channel rate allocation for Progressive transmission of images," IEEE Transactions on Communications, Vol. 51, No. 2, pp. 186-196, February 2003.

[8] P.G. Sherwood and K. Zeger, "Progressive image coding for noisy channels," IEEE Signal Processing Letters, Vol. 4, No. 7, pp. 189-191, July 1997.

[9] C. Lan, T. Chu, K.R. Narayanan, and Z. Xiong, "Scalable image and video transmission using irregular repeat-accumulate codes with fast algorithm for optimal unequal error protection," IEEE Transactions on Communications, Vol. 52, No. 7, pp. 1092-1101, July 2004.

[10] V.S. Sanchez and M.K. Mandal, "Robust transmission of JPEG2000 images over noisy channels," IEEE Transaction on Consumer Electronics, Vol. 48, No. 3, pp. 451-456, August 2002. 
[11] V. S. Sanchez and M.K. Mandal, “ Efficient channel protection for JPEG2000 bitstream," IEEE Transaction on Circuits and Systems for video technology, Vol. 14, No. 4, pp. 554-558, April 2004.

[12] J. Hagenauer, "Rate compatible punctured convolutional (RCPC) codes and their applications," IEEE Transaction on Communications, Vol. 36, pp. 389-400, April 1988.

[13] B.A. Banister, B. Belzer, and T.R. Fischer, "Robust image transmission using JPEG2000 and Turbo-codes," IEEE Signal Processing Letters, Vol.9, No.4, pp. 117119, April 2002.

[14] X. Pan, A. Cuhadar, and A.H. Banihashemi, "Combined source and channel coding with JPEG2000 and rate-compatible low-density parity-check (RC-LDPC) codes," IEEE Transactions on Signal Processing, Vol. 54, No. 3, pp. 1160-1164, March 2006.

[15] V. Chande and N. Farvardin, "Progressive Transmission of Images over memoryless noisy channels," IEEE Journal on Selected Areas in communications, Vol. 18, No.6, pp. 850-860, June 2000 .

[16] R. Hamzaoui, V. Sankovic, and Z. Xiong, "Rate-based versus distortion-based optimal joint source-channel coding," Proceedings of Data Compression Conference (DCC), pp. 63-72, April 2002.

[17] X. Pan, A.H. Banihashemi and A. Cuhadar, "A fast trellis-based rate-allocation algorithm for robust transmission of progressively coded images over noisy channel," IEEE Transactions on communications, Vol. 54, No. 1, pp. 1-6, January 2006.

[18] A. Kwasinski, V. Chande, and N. Farvardin, "Delay-constrained joint sourcechannel coding using incremental redundancy with feedback," IEEE Information Theory Workshop, pp. 283-286, March 4-April 2, 2003. 
[19] D.J. Costello Jr., J. Hagenauer, H. Imai, and S.B. Wicker, "Applications of errorcontrol coding," IEEE Transactions on Information Theory, Vol. 44, pp. 2531-2560, October 1998.

[20] V. Chande and N. Farvardin, "Image communication over noisy channels with feedback," International Conference on Image Processing, Vol. 2, pp. 540-544, October 1999.

[21] V. Chande, H. Jafarkhani, and N. Farvardin, "Joint source-channel coding of images for channels with feedback," IEEE Information Theory Workshop, San Diego, CA, pp. 50-51, February 1998.

[22] A.H. Banihashemi, and A. Hatam, "A distortion optimal rate allocation algorithm for transmission of progressive bitstreams over noisy channels", IEEE Data Compression Conference, pp. 376-376, March 2007.

[23] X. Pan A. H. Banihashemi, and A. Cuhadar, "Progressive transmission of images over fading channels using rate-compatible LDPC codes," IEEE Transactions on Image Processing, Vol. 15, No. 12, pp. 3627-3635, December 2006.

[24] Richard Bellman, Dynamic programming, Princeton University Press, Princeton, NJ, 1957.

[25] X. Pan, "Robust image transmission with rate-compatible low-density parity-check codes over noisy channels," Master thesis, Carleton, 2005.

[26] F. Etemadi, H. Yousefi'zadeh, and H. Jafarkhani, "A linear-complexity distortion optimal scheme for the transmission of packetized progressive bitstreams," IEEE Signal Processing Letters, Vol. 12, No. 5, pp. 365-368, May 2005.

[27] D.N. Rowitch and L.B. Milstein, "On the performance of Hybrid FEC/ARQ systems Using Rate Compatible Punctured Turbo (RCPT) Codes," IEEE Transactions on Communications, Vol. 48, No. 6, pp. 948-959, June 2000.

[28] Kakadu software implementation of the JPEG2000 standard [online]: http://www.kakadusoftware.com. 
[29] Jasper software implementation of the JPEG2000 standard [online]: http://www.ece.uvic.ca/ mdadams/jasper/

[30] J. Song and K.J. Ray Liu, "Robust progressive image transmission over OFDM systems using space-time block code," IEEE Transactions on Multimedia, Vol. 4, No. 3, pp. 394-406, September 2002.

[31] E. Steinbach, N. Farber, and B. Girod, "Standard compatible extension of H.263 for robust video transmission in mobile environments," IEEE Transactions on Circuits and System for Video technology, Vol. 7, No. 6, pp. 872-881, December 1997.

[32] X. Wang, J. Song, and K.J. Ray Liu, "Joint source and channel matching with channel estimation for robust image transmission over wireless channel," Vehicular Technology Conference, IEEE VTS 54 ${ }^{\text {th }}$, Vol. 1, pp. 428-432, Fall 2001. 